\title{
Finite-temperature coupled cluster: Efficient implementation and application to prototypical systems
}

Cite as: J. Chem. Phys. 152, 224104 (2020); https://doi.org/10.1063/5.0009845

Submitted: 03 April 2020. Accepted: 21 May 2020. Published Online: 09 June 2020

Alec F. White (D), and Garnet Kin-Lic Chan (D)

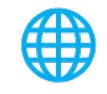

\section{Lock-in Amplifiers up to $600 \mathrm{MHz}$}

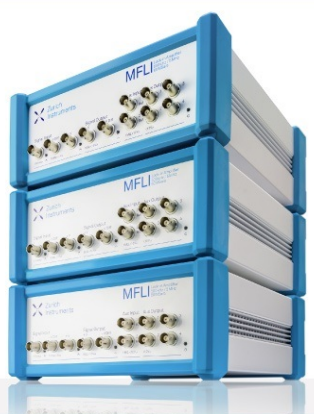




\title{
Finite-temperature coupled cluster: Efficient implementation and application to prototypical systems
}

\author{
Cite as: J. Chem. Phys. 152, 224104 (2020); doi: 10.1063/5.0009845 \\ Submitted: 3 April 2020 - Accepted: 21 May 2020 • \\ Published Online: 9 June 2020
}

Alec F. White ${ }^{\text {a) }}$ (D) and Garnet Kin-Lic Chan ${ }^{\text {b }}$

AFFILIATIONS

Division of Chemistry and Chemical Engineering, California Institute of Technology, Pasadena, California 91125, USA

a) Author to whom correspondence should be addressed: whiteaf@berkeley.edu

b) Electronic mail: gkc1000@gmail.com

\begin{abstract}
We discuss the theory and implementation of the finite temperature coupled cluster singles and doubles (FT-CCSD) method including the equations necessary for an efficient implementation of response properties. Numerical aspects of the method including the truncation of the orbital space and integration of the amplitude equations are tested on some simple systems, and we provide some guidelines for applying the method in practice. The method is then applied to the 1D Hubbard model, the uniform electron gas (UEG) at warm, dense conditions, and some simple materials. The performance of model systems at high temperatures is encouraging: for the one-dimensional Hubbard model, FTCCSD provides a qualitatively accurate description of finite-temperature correlation effects even at $U=8$, and it allows for the computation of systematically improvable exchange-correlation energies of the warm, dense UEG over a wide range of conditions. We highlight the obstacles that remain in using the method for realistic $a b$ initio calculations on materials.
\end{abstract}

Published under license by AIP Publishing. https://doi.org/10.1063/5.0009845

\section{INTRODUCTION}

An $a b$ initio description of the thermal properties of molecules and materials remains a significant challenge. In many cases, experimental temperatures are so small relative to the lowest energy electronic excitations that electronic temperature can be assumed to be effectively zero. However, there are cases where this assumption is not justified. Some examples include

1. warm, dense matter,

2. the low-energy phases of correlated materials, and

3. metallic systems.

In these systems, the electronic temperature cannot be ignored, and how best to incorporate thermal effects into computational methods for treating electron correlation is an open question.

In warm, dense matter, the thermal effects are comparable in magnitude to the effects of electron correlation. ${ }^{1,2}$ Conditions like this occur in planetary cores ${ }^{3}$ and can be realized in the laboratory with high intensity lasers. ${ }^{4,5}$ Finite temperature density functional theory (DFT) coupled with molecular dynamics (MD) for the nuclei is the most practical computational method for such systems. ${ }^{6-8}$ However, the parameterization of finite temperature density functionals is a non-trivial problem, ${ }^{9-11}$ and a variety of quantum Monte Carlo (QMC) methods have been developed with the goal of providing an accurate treatment of finite-temperature fermionic systems. $^{12-18}$ In this context, the warm, dense uniform electron gas (UEG) has become an important system both as a benchmark for new methods and as an ingredient in the parameterization of finite temperature density functionals. ${ }^{1}$

The rich electronic phases of correlated materials also require a treatment of electron correlation at finite temperature. Here, the low energy excitations typically involve the spin degrees of freedom, and thus, phase transitions can occur on the emergent exchange coupling temperature (or lower) scales. Theoretical work has largely focused on model systems such as the Hubbard model. ${ }^{30-34}$ For such lattice problems, a variety of methods including dynamical mean field theory (DMFT), ${ }^{35,36}$ the dynamical cluster approximation (DCA), ${ }^{37,38}$ and finite temperature extensions to the density matrix renormalization group (DMRG) $)^{39-41}$ are commonly used. 
In $a b$ initio calculations on metals, the lack of a bandgap creates numerical difficulties that can be solved by including a finite electronic temperature. DFT often offers a good description, and it is common practice to use a thermal smearing of the electron density to ease convergence of the Kohn-Sham equations. ${ }^{42,43}$ Explicit treatment of electron correlation in metals beyond density functional theory is less common, though GW theory has been applied to metals. ${ }^{44} A b$ initio DMFT has been used to study correlated metallic systems, especially those which undergo a low-temperature phase transition due to electron correlation (see Sec. IV of Ref. 36 for a review).

Problems like these have spurred recent interest in extending $a b$ initio electronic structure methods to the case of finite electronic temperatures. The simplest methods in this hierarchy are thermal mean-field theories, Hartree-Fock (HF) theory, ${ }^{45}$ or DFT. ${ }^{46}$ The goal is to develop hierarchies that mirror those at zero temperature and approach the thermal full configuration interaction (FCI) limit with polynomial scaling approaches. Examples include finite temperature extensions of perturbation theory, ${ }^{48,49}$ configuration interaction (CI), ${ }^{50}$ Green's function methods, ${ }^{51-53}$ or coupled cluster (CC) theory.

The coupled cluster method is the method of choice for highaccuracy, ground-state, quantum chemistry calculations, ${ }^{58-64}$ and we believe it to be a promising method for finite temperature calculations as well. The first polynomial-scaling finite temperature generalization of coupled cluster theory was the thermal cluster cumulant (TCC) theory of Mandal and co-workers. ${ }^{54,65-68}$ Recently, there has been renewed interest in finite-temperature coupled cluster methods. Hermes and Hirata suggested a coupled cluster doubles method based on their "renormalized" perturbation theory, which is simple to implement but lacks a rigorous justification. White and Chan presented a finite-temperature extension of CCSD $(\text { FT-CCSD })^{55}$ that can be viewed as a time-dependent diagrammatic derivation and computational realization of TCC theory. Independently, Hummel published a finite-temperature randomphase approximation (linearized, direct coupled cluster doubles) method for periodic solids, which uses the same conceptual framework. Recently, Harsha et al. derived a finite temperature coupled cluster theory based on the thermofield formalism. ${ }^{57}$ Coupled cluster methods for the dynamics of finite temperature systems driven out of equilibrium have also been the subject of several recent studies. ${ }^{69-71}$ Despite all this development, many practical questions remain unanswered, and it is the goal of this work to address such questions.

Working within the FT-CCSD formalism presented in Ref. 55, we will clarify several aspects of the theory and present the equations necessary for an efficient implementation of FT-CCSD including a response treatment of properties. In Sec. III, we will discuss the numerical and computational aspects of the method in the context of some simple benchmark calculations. In Sec. IV, we apply the method to several finite temperature systems. The 1D Hubbard model allows us to compare to the exact results for different values of the onsite repulsion, and we find that FT-CCSD performs well even for $U=8$, a relatively large value of the onsite repulsion. We present FT-CCSD calculations of the UEG exchangecorrelation energy at finite temperature with particular emphasis on the potential of FT-CC methods to provide consistent, systematically improvable results over a wide range of temperatures and densities. Finally, some simple $a b$ initio calculations on periodic solids serve to demonstrate both the potential of the method and the difficulties we face in $a b$ initio calculations at a finite electronic temperature.

\section{THEORY}

Here, we review and expand on the theory presented in Ref. 55. The theory is, in a fundamental sense, identical to the thermal cluster cumulant (TCC) theory of Mandal and co-workers, ${ }^{54,65-68}$ but our focus is on using the FT-CCSD theory presented in Ref. 55 as a computational tool.

\section{A. The FT-CC equations: Integral and differential forms}

The FT-CC contribution to the grand potential is determined from an integration in imaginary time,

$$
\Omega_{C C}=\frac{1}{\beta} \int_{0}^{\beta} d \tau \mathrm{E}[\mathbf{s}(\tau)]
$$

where $\beta$ is the inverse temperature, $\mathbf{s}$ is a vector of FT-CC amplitudes, and the kernel, E, is local in imaginary time and given in Eq. (A8).

The FT-CC equations can be derived directly from diagrammatics as in Ref. 55 or from the thermally normal-ordered ansatz of TCC. The amplitude equations are non-linear and, in integral form, are given by

$$
s_{v}(\tau)=-\int_{0}^{\tau} d \tau^{\prime} e^{\Delta_{v}\left(\tau^{\prime}-\tau\right)} S_{v}\left[\mathbf{s}\left(\tau^{\prime}\right)\right]
$$

The index, $v$, runs over the amplitudes that are typically truncated at some excitation level. Here, the $S$ kernel is local in imaginary time and is given in Appendix A for the case of finite-temperature coupled cluster singles and doubles (FT-CCSD). $\Delta_{v}$ is the difference of orbital energies associated with the $v$ th excitation. In Ref. 55, we chose to define the amplitudes, $s$, such that the occupation numbers were associated with each line appearing "above" the interaction diagrammatically. Here, we adopt a slightly different convention where the occupation numbers are split symmetrically. For example, at first order, the definition of $s_{i}^{a}(\tau)$ differs between Ref. 55 and this work,

$$
\begin{aligned}
\text { Ref. 55: } & f_{a i}\left(1-n_{a}\right) . \\
\text { This work: } & f_{a i} \sqrt{n_{i}\left(1-n_{a}\right)},
\end{aligned}
$$

where $n$ is the Fermi-Dirac occupation number, $f$ is the finite temperature Fock matrix, and orbital indices are indicated by $i$ and $a$. The appropriate modifications to the amplitude and energy equations are shown in Appendix A. This modification changes neither the theory nor the results, but it allows us to form effective integrals that retain the symmetry of the underlying integrals, thereby decreasing the memory usage in practical calculations. Furthermore, it leads to a more symmetric treatment of the $\lambda$ amplitudes so that both quantities remain similar in magnitude in the low-temperature limit. 
In order to efficiently compute properties, we define a variational Lagrangian,

$\mathcal{L} \equiv \frac{1}{\beta} \int_{0}^{\beta} d \tau \mathrm{E}(\tau)+\frac{1}{\beta} \int_{0}^{\beta} d \tau \lambda^{v}(\tau)\left[s_{v}(\tau)+\int_{0}^{\tau} d \tau^{\prime} e^{\Delta_{v}\left(\tau^{\prime}-\tau\right)} S_{v}\left(\tau^{\prime}\right)\right]$.

Note that this definition differs by a minus sign from that given in Ref. 55. This sign convention does not change the results but makes the $\lambda$ equations more closely resemble those of the groundstate theory. The $\lambda$ amplitudes are defined by the condition that $\mathcal{L}$ is stationary with respect to variations of the $s$ amplitudes, which leads to a linear equation,

$$
\lambda^{v}(\tau)=-\mathrm{L}[\mathbf{s}(\tau), \tilde{\lambda}(\tau)] .
$$

We define the quantity, $\tilde{\lambda}$, as

$$
\tilde{\lambda}^{v}(\tau) \equiv \int_{\tau}^{\beta} d \tau^{\prime} e^{\Delta_{v}\left(\tau-\tau^{\prime}\right)} \lambda^{v}\left(\tau^{\prime}\right)
$$

Given these integral equations [Eqs. (2) and (6)], one can easily obtain differential equations for $s$ and $\tilde{\lambda}$ directly,

$$
\begin{aligned}
& \frac{d s_{v}}{d \tau}=-\left\{\Delta_{v} s_{v}(\tau)+\mathrm{S}_{v}[\mathbf{s}(\tau)]\right\}, \\
& \frac{d \tilde{\lambda}_{v}}{d \tau}=\left\{\Delta_{v} \tilde{\lambda}_{v}(\tau)+\mathrm{L}_{v}[\mathbf{s}(\tau), \tilde{\lambda}(\tau)]\right\} .
\end{aligned}
$$

These equations, in integral [Eqs. (2), (6), and (7)] or differential [Eqs. (8) and (9)] form, are described in more detail in Appendix A for the specific case of FT-CCSD. Once the $\lambda$ amplitudes have been computed, properties can be evaluated by computing the partial derivatives of the Lagrangian. In Sec. II D, we will show how these derivatives, including the response of the reference orbital energies, can be computed by contracting the basis representation of an operator with response densities.

\section{B. Choice of reference}

Like in zero temperature coupled cluster theory, the choice of reference orbitals will have some effect on the energy and properties. Unlike the ground-state theory, the choice of orbital energies will also have an effect. In other words, for a given choice of orbitals, the relative partitioning of the energy between between zeroth and first order will matter at finite temperature even though it does not at zero temperature. This difference is most easily conceptualized within the TCC formulation, which uses a thermally normal ordered ansatz. At zero temperature, partitioning the orbitals into an occupied and virtual space defines entirely the normal-ordering with respect to that reference,

$$
N[A B C \ldots]_{T=0}=A B C \ldots-\langle A B C \ldots\rangle_{T=0} .
$$

This is because the expectation value in the zero temperature reference is determined entirely by the choice of occupied space. However, at some finite temperature $\left(T=T_{0}\right)$, the normal-ordering depends on the occupations explicitly,

$$
N[A B C \ldots]_{T=T_{0}}=A B C \ldots-\langle A B C \ldots\rangle_{T=T_{0}} .
$$

This is because the thermal average will depend on the occupation numbers of the states in question, which, in turn, are functions of the non-interacting, single-particle energies.

This means that we must always be careful to specify the reference energies as well as orbitals used for a particular calculation since it will affect the final answer. There are many possible choices of reference orbitals and energies, and some aspects of the choice of reference have been described by Sanyal et al.

\section{Numerical integration and propagation in imaginary time}

In practice, the imaginary-time integral to determine the free energy must be done by numerical quadrature,

$$
\int_{0}^{\beta} I(\tau) d \tau \approx \sum_{x} g^{x} I\left(\tau_{x}\right) .
$$

The values of amplitudes at some finite set of $n_{g}$ points are stored, and the tensor $g$ contains the quadrature weights. If the integral form of the equations is used [see Eqs. (2), (6), and (7)], then the amplitudes are determined by solving an integral equation of the form

$$
s\left(\tau_{y}\right) \sim \int_{0}^{\tau_{y}} I(\tau) d \tau \approx \sum_{x} G_{y}^{x} I\left(\tau_{x}\right),
$$

where $G$ is a tensor of quadrature weights and the integrand $I$ depends on the amplitudes. On the other hand, if the differential form of the equations is used [see Eqs. (8) and (9)], then the amplitudes are propagated like

$$
s\left(\tau_{y}\right)=s\left(\tau_{y-1}\right)+\Delta s,
$$

where the step, $\Delta s$, is determined either from a differential equation integrator such as a Runge-Kutta, ${ }^{73,74}$ Bashforth and Adams, ${ }^{75,76}$ or Crank-Nicolson ${ }^{77}$ method. There is a relationship between the integral and differential form of the equations in that any integral method defined by a set of quadrature rules encoded in $G$ should be equivalent to some, generally non-trivial, integrator. If $G$ has nonzero diagonal entries, then the associated integral iteration is equivalent to an implicit propagation scheme, such as Crank-Nicolson, and otherwise it will be equivalent to an explicit propagation scheme, such as fourth order Runge-Kutta.

\section{Response properties}

Properties in FT-CC theory are best computed from the response of the grand potential to a perturbation. This is most easily accomplished by computing the analytic derivatives of the Lagrangian presented in Eq. (5). The $\lambda$ amplitudes are computed such that this Lagrangian is stationary with respect to variations in the amplitudes, so we need not consider the response of the amplitudes directly, but there are still several types of responses that must be considered. We will first consider the derivative with respect to a parameter $\alpha$, where $\alpha$ represents the coupling to some operator $X$. In this case, there are three types of terms:

1. Terms resulting from the explicit dependence of the Hamiltonian on $\alpha$.

2. Terms resulting from the dependence of the occupation numbers and orbital energies on $\alpha$. 
3. Terms resulting from the dependence of the orbitals themselves on $\alpha$.

Unlike in the ground state coupled cluster, the orbital energies and the one-electron part of the perturbation appear separately in the Lagrangian. This means that properties will depend on the relative partitioning of $X$ into a part that is included in the orbital energies and a part that appears as part of the perturbation, e.g., for a one-electron $X$,

$$
X_{p q}=X_{q}^{(0)} \delta_{p q}+X_{p q}^{(1)} .
$$

Terms of type 1 are the simplest, and they may be efficiently computed by tracing $X^{(1)}$ with the unrelaxed, normal-ordered FT-CCSD 1-RDM, $\gamma_{N}$ (or the 2-RDM $\Gamma_{N}$ for two-electron properties), as described in Appendix B. We use the subscript $N$ to indicate that these densities represent the response only to the thermally normal ordered part of the operator. Terms of type 2 can be incorporated by tracing a diagonal matrix, $d$, with $X^{(0)}$. The computation of this quantity is also described in Appendix $\mathrm{B}$. The incorporation of the response of the orbital energies and occupation numbers is crucial to obtaining a density matrix that has a trace equal to the electron number computed as $-\partial \Omega / \partial \mu$. The orbital response (type 3 ) must be included to compute fully relaxed properties, and it is possible to also incorporate this into a fully relaxed density matrix. In this work, we ignore this contribution for several reasons. First, in most cases, we use zero temperature orbitals, which means that there will be no orbital response contribution to the energy, entropy, or number of electrons. Furthermore, for the UEG, this term is rigorously zero because the form of the orbitals is fixed by the translation invariance of the system. Finally, we suspect that, as in zero-temperature CCSD, the orbital contribution to most properties is small, though this should ultimately be verified numerically. The entropy can be computed from the derivative with respect to $\beta$ for which there are additional terms that we must consider

4. terms arising from the explicit dependence of the Lagrangian on $\beta$,

5. terms arising from the dependence of the quadrature weights on $\beta$, and

6. terms arising from the positions of the grid points that depend on $\beta$.

Terms of type 4 are simply proportional to the value of the Lagrangian itself [Eq. (B35)]. Terms of type 5 are related ultimately to the dependence of the integration limits on $\beta$. Terms of type 6 can also be computed for a given discretization, though these terms will vanish in the limit of a dense grid [Eq. (B38)]. Precise equations for all these terms are given in Appendix B.

\section{BENCHMARKS}

In this section, we will use some simple benchmarks to suggest an answer to several practical questions. How severe an approximation is the truncation of the amplitudes based on small occupation numbers? What types of grids are most effective and how many grid points are necessary to obtain a desired accuracy? What computational resources are required to perform a given calculation? In exploring these questions, we will focus on two small systems: the beryllium atom in a minimal basis (STO-3G) at fixed $\mu(\mu=0)$ and the 14 electron, unpolarized UEG in a basis of 33 plane wave orbitals at a fixed average number of electrons, i.e., $\mu$ is adjusted such that $\langle N\rangle=14$.

\section{A. Restricted occupied and virtual spaces}

One of the simplest ways to reduce the cost of FT-CCSD is to allow nonzero amplitudes only when the "occupied" ("virtual") indices are associated with orbitals that have particle (hole) occupation number greater than some threshold. In general, such a truncation will lead to approximate results, and we must ask what error is incurred and what kinds of thresholds are acceptable.

Although it is tempting to assume that the contribution to the free energy due to excitation from an orbital with occupation $n_{i}$ is proportional to $n_{i}$, this is unfortunately not the case, and we must be careful when truncating the excitation space in this way. As a rough estimate, consider the second order contribution to the grand potential due a one-particle matrix element $v_{a i}$,

$$
\Omega_{a i}^{(2)}=\frac{1}{\beta} n_{i}\left(1-n_{a}\right)\left|v_{a i}\right|^{2}\left[\frac{\beta}{\varepsilon_{i}-\varepsilon_{a}}+\frac{1-e^{\beta\left(\varepsilon_{i}-\varepsilon_{a}\right)}}{\varepsilon_{i}-\varepsilon_{a}}\right] .
$$

For the purpose of this analysis, we will assume, without loss of generality, that $\mu=0$. Consider the case where $\beta$ and/or $\varepsilon_{i}$ are large such that

$$
n_{i} \sim e^{-\beta \varepsilon_{i}} .
$$

If we furthermore assume that $v_{a i}$ and $\left(1-n_{a}\right)$ are of order 1 and $\varepsilon_{a}$ is small, then we can extract the asymptotic behavior of the second order expression, and we find that

$$
\Omega_{a i}^{(2)} \sim \frac{1}{\beta \varepsilon_{i}^{2}}
$$

This would seem to suggest that, as a rigorous threshold, one should assume an error that goes like the natural log of the occupation numbers, $\left(-\varepsilon_{i} \ln n_{i}\right)^{-1}$, and not the occupation numbers themselves. This behavior is shown in Fig. 1 for the beryllium atom. Note that with $\mu$ being fixed at $\mu=0$, the number of electrons will vary from about $N \approx 4.53$ at $T \approx 100000 \mathrm{~K}$ to $N=4$ as $T \rightarrow 0$. This figure clearly shows that the error incurred by truncating the cluster amplitudes is some polynomial in the inverse temperature even though the occupation numbers themselves decay exponentially. Despite this fact, we have observed that a threshold of approximately $1 \times 10^{-30}$ is sufficient to guarantee errors of $<1 \mathrm{meV}$ per electron relative to the full FT-CCSD. This corresponds to the region of Fig. 1 where the truncated occupation (lower panel) is $<1 \times 10^{-30}$. In general, this may be system dependent, and it is always prudent to examine the convergence of relevant properties with respect to this threshold.

\section{B. Numerical integration and propagation schemes}

Efficient FT-CCSD calculations are critically dependent on the numerical quadrature used to compute the grand potential and the integral or differential scheme used to solve for the amplitudes. In this section, we will discuss two questions: 

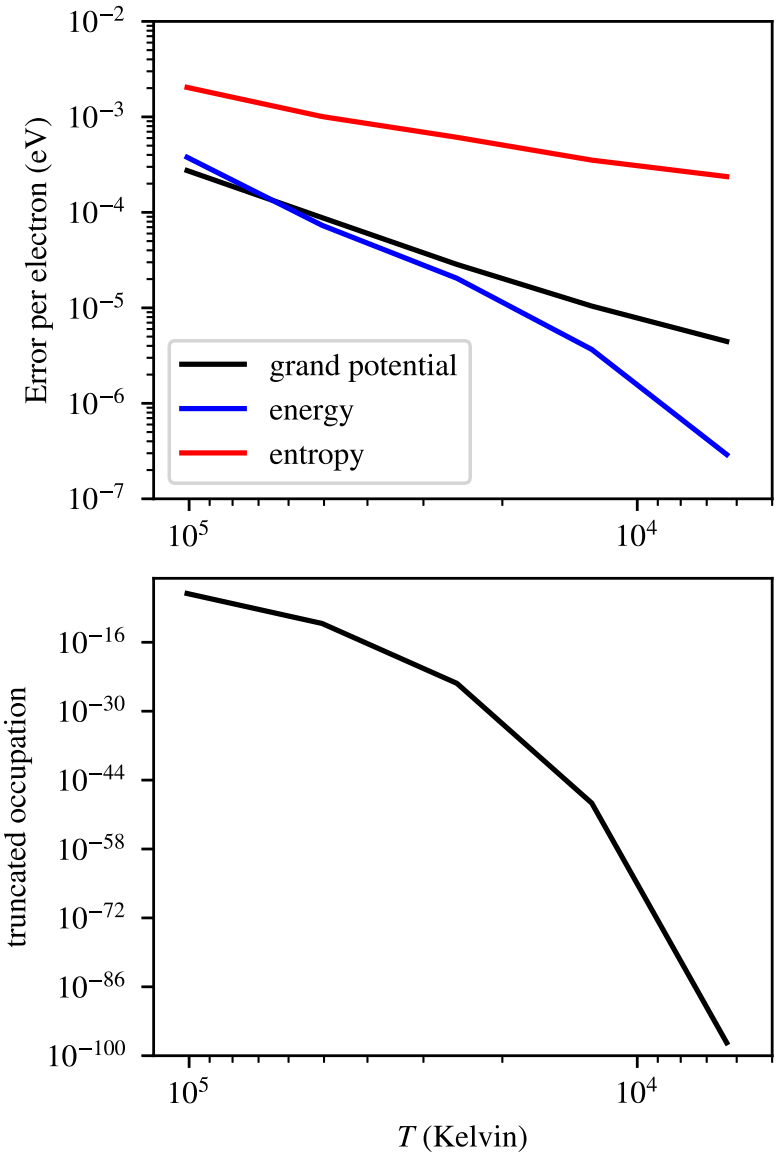

FIG. 1. Upper panel: error per electron due to truncating the "virtual" space of the minimal basis (STO-3G) Be atom so as not to include the lowest energy (1s) orbital. (Note that in the finite-temperature theory, the virtual space includes all orbitals, the virtual labels serving only to indicate the time direction of the propagator associated with the orbital.) The lower panel shows the hole occupation, $1-n_{a}$, of this orbital. While the occupation decreases exponentially with temperature, the corresponding contribution to the properties decreases like some polynomial in temperature.

1. How does the error depend on the number of grid points for some simple numerical schemes?

2. How many grid points are typically required?

One has great freedom in choosing a propagation scheme and numerical integration scheme, and we cannot claim that the methods we use in this work are optimal. We believe that the best choice will ultimately be an adaptive scheme, but this is beyond the scope of this work.

In this work, we use a quadrature generated by Simpson's rule $^{78}$ and use either the implicit integral method generated by the same Simpson's rule or an explicit Runge-Kutta (RK) propagator to compute the amplitudes. Even though we use the same grid for the quadrature that determines the grand potential and the integrator that determines the amplitudes, these are really separate sources of numerical error. In Fig. 2, we show the error in the
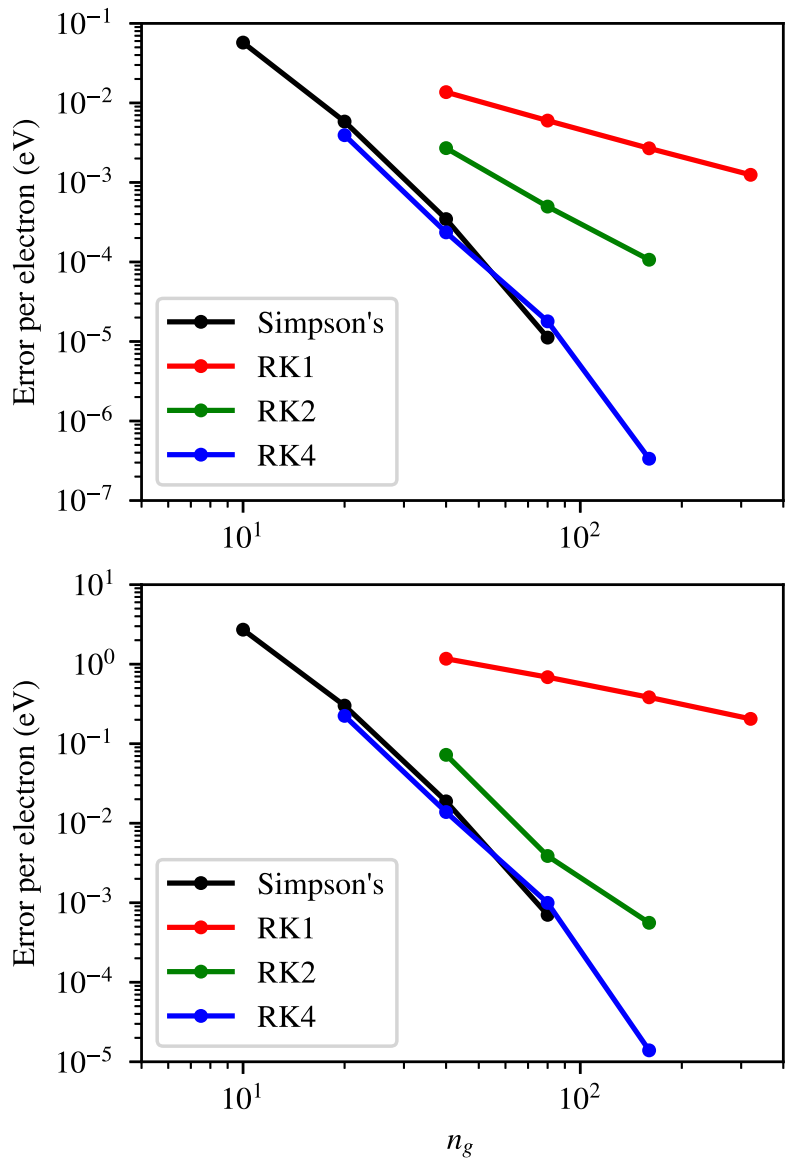

FIG. 2. The error per electron in the exchange-correlation energy (top) and exchange-correlation entropy (bottom) for different integrators as a function of the number of grid points. The polynomial behavior of the error in the grid spacing matches the theoretical expectation (see Table I).

exchange-correlation energy and exchange-correlation entropy of the 14 electron unpolarized UEG in 33 plane-wave orbitals. The density is characterized by $r_{s}=4$, and the reduced temperature is $\theta=0.125$. Note that in all cases, the numerical error is controllable. The error in the entropy is larger than the error in the energy, and this is to be expected because

$$
S=-\beta(\Omega-E-\mu N) .
$$

Therefore, any error in the energy should be magnified in the entropy by a factor of the inverse temperature. This is consistent with the behavior observed in Fig. 1. One must be cautious in using Fig. 2 to suggest the "best" numerical method. Although Simpson's rule integrator may provide the smallest error for a given number of grid points, this implicit method requires the iterative solution of a non-linear equation at each step and is therefore considerably more expensive. We will return to this point in Sec. III C.

The theoretical and observed behavior of the numerical error is summarized for different methods in Table I. This confirms that 
TABLE I. Theoretical vs observed asymptotic error with respect to the step size, $h=1 / n_{g}$. The first column indicates the method used to compute the amplitudes, the second indicates the asymptotic behavior of the error due to the quadrature used to compute the grand potential and its derivatives, and the third column shows the asymptotic behavior of the error due to the numerical solution of the amplitudes themselves. The final two columns show the behavior observed in Fig. 2 for the error in the exchange-correlation energy and entropy, respectively.

\begin{tabular}{lcccc}
\hline \hline Solver & Int. error & Amp. error & Obs. $\left(E_{x c}\right)$ & Obs. $\left(S_{x c}\right)$ \\
\hline Simpson's & $h^{4}$ & $h^{4}$ & $h^{4.1}$ & $h^{4.0}$ \\
RK1 & $h^{4}$ & $h^{1}$ & $h^{1.2}$ & $h^{0.8}$ \\
RK2 & $h^{4}$ & $h^{2}$ & $h^{2.4}$ & $h^{2.8}$ \\
RK4 & $h^{4}$ & $h^{4}$ & $h^{4.4}$ & $h^{4.6}$ \\
\hline \hline
\end{tabular}

these numerical methods are behaving as expected and provide an answer to question (1). Furthermore, the clear asymptotic behavior of the numerical error allows us to estimate the numerical error in a given calculation and extrapolate the dense grid limit if desired. The deviations of the asymptotic error from its expected behavior in some cases may be due to the number of electrons not being sufficiently converged. For this system, we fixed the number of electrons separately for each number of grid points to better than $1 \times 10^{-4}$. In practice, the answer to question (2) can be obtained by monitoring the change in properties of interest as the number of grid points is increased.

\section{Timings and computational considerations}

We must also consider the computational aspects of these calculations. For the numerical integration, we can use either an explicit or implicit integrator in the solution of the amplitude equations. Implicit methods will generally be more accurate and more stable at the cost of iteratively solving a non-linear equation at each grid point. This trade-off is illustrated in Table II, which suggests that explicit methods will usually be cheaper, though implicit methods may be preferable in some cases.

In particular, we have observed that at lower temperatures, the differential equations can become "stiff." In such cases, the step-size necessary to stably integrate the equations with an explicit method may be impractically small and an implicit integrator may be more efficient.

TABLE II. Minimum number of grid points (given calculations for $n_{g}=20,40,80,160$, 320) necessary to obtain sub-millivolt error per electron in the exchange-correlation energy for the 14 electron UEG system $\left(r_{s}=4, \theta=0.125\right)$ and the time of that calculation. All calculations were performed on a single 28 core node.

\begin{tabular}{lcc}
\hline \hline Method & $n_{g}$ & Time (s) \\
\hline Simpson's & 40 & 4054 \\
RK1 & 320 & 5720 \\
RK2 & 80 & 2447 \\
RK4 & 40 & 2128 \\
\hline \hline
\end{tabular}

ALGORITHM 1. Solve for the amplitudes using the differential form of the equations. The key step is line 4 where either an explicit integrator (such as RK4) or an implicit integrator (such as the Crank-Nicolson method) is used to find the step.

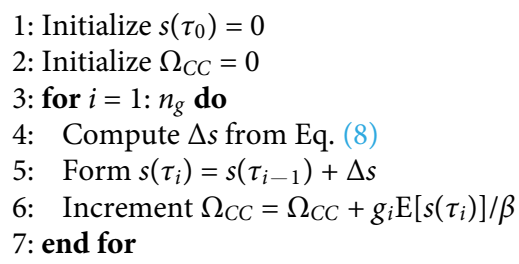

ALGORITHM 2. Solve for the amplitudes using the integral form of the equations. The key step is line 4 where $s\left(\tau_{i}\right)$ is solved from Eq. (2) discretized, as shown in Eq. (13). Depending on the form of the quadrature, this may or may not require the iterative solution of a system of non-linear equations.
1: Initialize $s\left(\tau_{0}\right)=0$
2: for $i=1: n_{g}$ do
3: Compute $S\left[s\left(\tau_{j}\right)\right]$ for $j \leq i$
4: Compute $s\left(\tau_{i}\right)$ from Eq. (2)
5: end for
6: Compute $\Omega_{c c}$ from Eq. (1)

Although the differential and integral forms of the amplitude equations do not differ conceptually, they suggest slightly different algorithms. The algorithm that mirrors the differential form of the algorithm is described in Algorithm 1. The algorithm that follows the integral form of the equations is given in Algorithm 2. In both cases, the most expensive step is the evaluation of the S kernel [Eqs. (A15) and (A16) for CCSD]. The number of times that this kernel must be evaluated depends on the specific integrator or quadrature.

The computational scaling of FT-CCSD is asymptotically the same as for ground-state CCSD, but the prefactor is considerably larger due to the number of grid points and the fact that there is no distinction between "occupied" and "virtual" orbital spaces. The additional memory cost due to the grid points can be ameliorated by using disk storage, as shown in Table III. Technical improvements, such as distributed memory parallelization, are necessary to improve the performance further.

TABLE III. Scaling of disk storage, memory, and cpu time for the fully incore and diskbased implementations of FT-CCSD. $N$ indicates the number of orbitals and $n_{g}$ the number of grid points.

\begin{tabular}{llll}
\hline \hline Method & Disk & Mem. & cpu \\
\hline Incore & $\ldots$ & $n_{g} N^{4}$ & $n_{g} N^{6}$ \\
Disk & $n_{g} N^{4}$ & $N^{4}$ & $n_{g} N^{6}$ \\
\hline \hline
\end{tabular}




\section{APPLICATIONS}

In order to demonstrate some features of the FT-CCSD method, we will now apply it to several prototypical systems. The differential form of the equations (Algorithm 1) is used in all cases, and the numerical error due to imaginary time discretization is converged to better than $0.1 \mathrm{~m} E_{\mathrm{h}}$ or $0.0001 t$ for the Hubbard model. For the UEG and $a b$ initio Hamiltonians, $\mu$ must be adjusted to obtain the desired number of electrons, $N$. This simple one-dimensional root finding problem is solved by the secant method and typically takes no more than five iterations to converge.
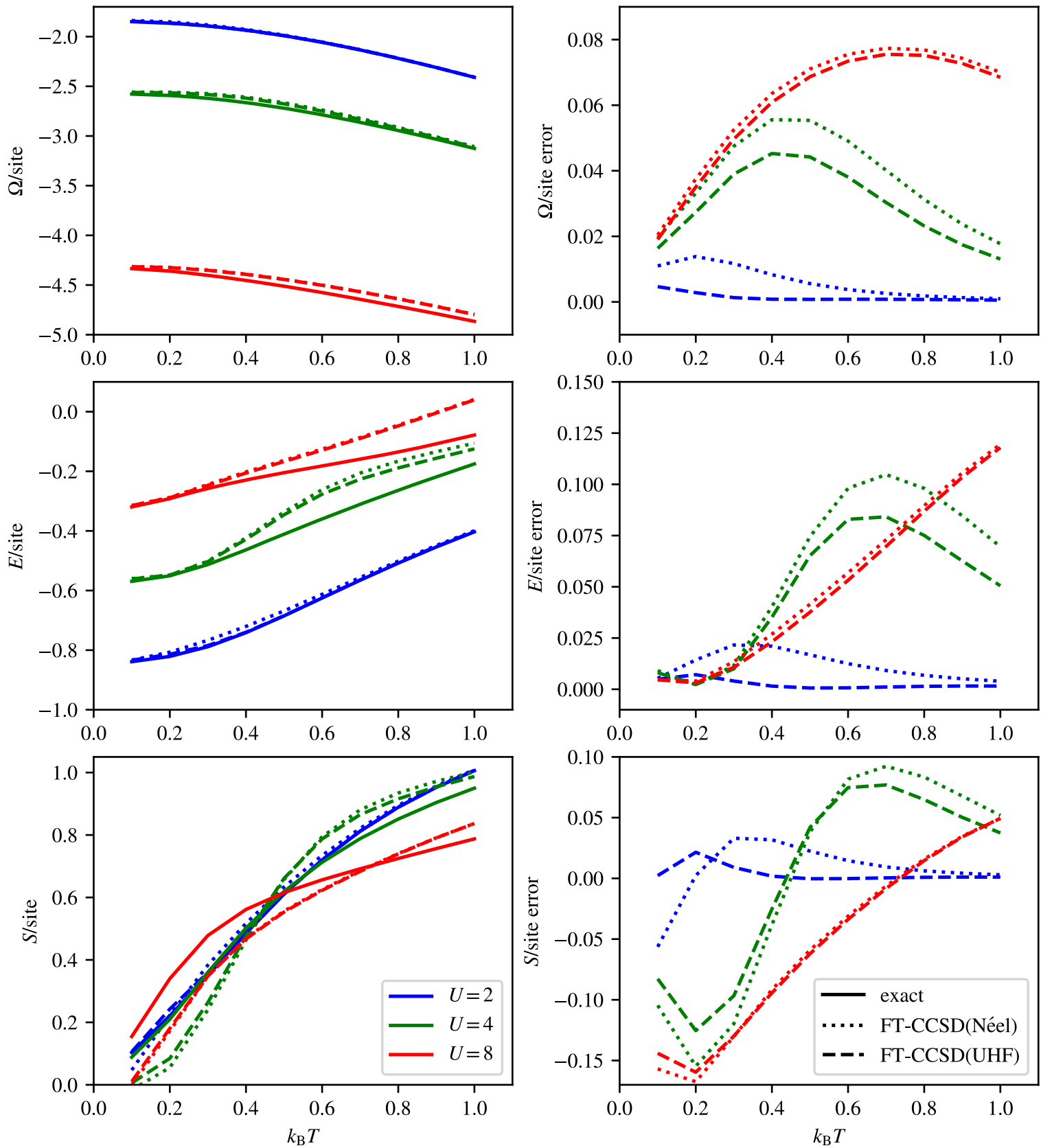

FIG. 3. The grand potential per site (first row), energy per site (second row), and entropy per site (third row) as a function of temperature for the $1 \mathrm{D}$ Hubbard model with $U=2$ (blue), $U=4$ (green), and $U=8$ (red). All quantities are given in units of the hopping, $t$. 
For the Hubbard model and the warm-dense UEG, no truncation of the orbital space was employed. For calculations on $a b$ initio Hamiltonians, a threshold of $1 \times 10^{-30}$ was used to truncate the orbital space at lower temperatures, as described in Sec. III A. For each system, we verified that the results were not sensitive to this truncation.

\section{A. The Hubbard model}

First, we consider the one-dimensional (1D) Hubbard model, ${ }^{30}$ an exactly solvable model of strong correlation. The one-band, 1D Hubbard model is given by the Hamiltonian,

$$
H=-t \sum_{i \sigma}\left(a_{i, \sigma}^{\dagger} a_{i+1, \sigma}+a_{i+1, \sigma}^{\dagger} a_{i, \sigma}\right)+U \sum_{i} n_{i \uparrow} n_{i \downarrow},
$$

where $i$ runs over the sites of a one-dimensional lattice and $\sigma$ runs over the spin states of a spin-1/2 particle. The equilibrium properties of this model at finite temperature in the thermodynamic limit can be found exactly via the Bethe ansatz. This provides us with an opportunity to evaluate the strengths and weaknesses of FT-CCSD by comparing to an exact result for different values of the onsite repulsion $U / t$.

In Fig. 3, we show the exact and FT-CCSD grand potential, energy, and entropy per site for the 1D Hubbard model with periodic boundary conditions at half filling $(\mu=U / 2)$. The FTCCSD results are taken from a 32 site lattice, which is very close to the thermodynamic limit for the parameters considered here. FT-CCSD results from a Néel state reference and from a zerotemperature unrestricted Hartree-Fock (UHF) reference are shown to highlight the effect of different references. In both cases, the reference non-interacting system is defined by the diagonal entries of the zero-temperature Fock matrix. The exact results are computed via the Bethe ansatz. ${ }^{79}$ In all cases, even for the relatively strongly correlated case of $U=8$, FT-CCSD provides qualitatively correct results. The agreement with the exact result is better for smaller $U$ as we might expect from the performance of ground state CCSD on the Hubbard model. ${ }^{34,80,81}$ Although both sets of reference orbitals lead to FT-CCSD results with a similar level of accuracy, the optimized UHF orbitals clearly provide a better starting point. The difference between UHF and Néel orbitals gets smaller at larger $U$ where the UHF orbitals more closely resemble a Néel state.

In the first column, we plot the exact results (solid line) and FT-CCSD results with a Néel state reference (dotted line) and a UHF reference (dashed line). In the second column, the error in the FT-CCSD results is plotted. The entropy curves are also plotted in Appendix $\mathrm{C}$ for clarity.

The price that must be paid for coupled cluster calculations on strongly repulsive systems like this is artificial symmetry breaking in the reference orbitals. Both references (Néel and UHF) break spin symmetry, and this symmetry cannot be fully restored by FTCCSD, as shown in Fig. 4 where we plot the staggered magnetization per site in the FT-CCSD one-particle reduced density. The exact solution to this problem exhibits no long-range antiferromagnetic order as a consequence of the Mermin-Wagner theorem. This artificial symmetry breaking in the FT-CCSD density is not a serious issue if one is interested in just the energy or grand potential (see Fig. 3), but it will likely obscure certain types of
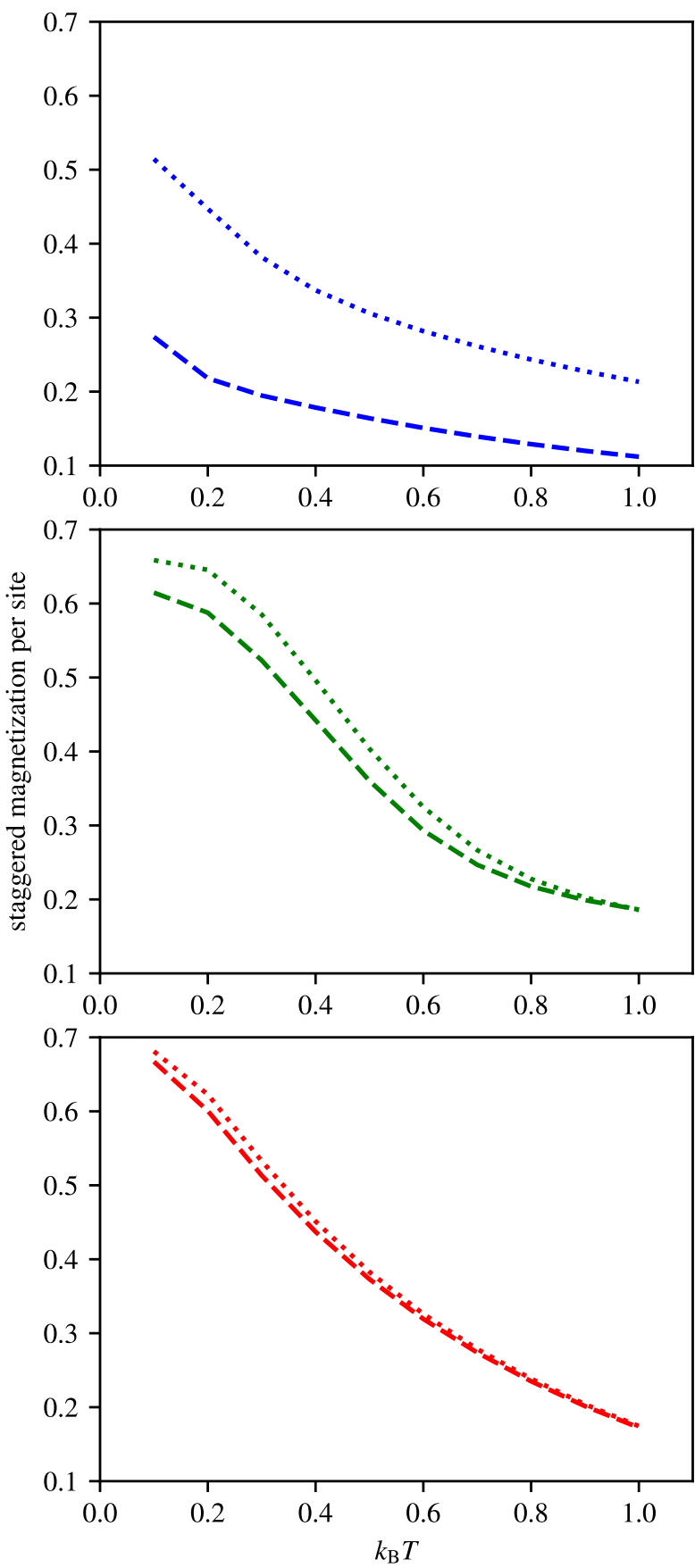

FIG. 4. The staggered magnetization in the FT-CCSD 1-particle reduced density matrix as a function of temperature for the 1D Hubbard model with $U=2$ (top, blue), $U=4$ (middle, green), and $U=8$ (bottom, red). Results for both Néel state reference (dotted line) and UHF reference (dashed line) are shown.

phase transitions. The systematic underestimation of the entropy at low temperatures shown in row 3 of Fig. 3 (and shown more clearly in Fig. 12) is also related to this artificial symmetry breaking since the configuration obtained by flipping all the spins of the 
reference is not well-described and cannot properly contribute to the entropy.

It is encouraging that FT-CCSD provides qualitatively correct results even for a relatively strongly correlated case of $U=8$. However, the symmetry-broken references required to obtain these results suggest that FT-CCSD would not be appropriate for describing a phase transition, such as the Néel transition in the threedimensional Hubbard model, which is governed by a spontaneous breaking of spin symmetry.

\section{B. The warm dense UEG}

The warm, dense UEG has been the focus of much work within the quantum Monte Carlo community with the focus being the accurate computation of the exchange-correlation energy. ${ }^{19-21,23-29,83,84}$ The 66 electron unpolarized and 33 electron polarized UEGs are the most commonly considered finite-size models. The work in this area is best summarized in Ref. 29. Our interest in the UEG is twofold: we compare to QMC results where accurate QMC results are available, and we evaluate the potential of FT-CC methods to provide the results for some sets of parameters where reliable QMC calculations are more difficult. In particular, note Figs. 18-20 of Ref. 29 where the state-of-the-art QMC calculations on these systems are summarized. We compare to the following finite temperature QMC methods: configuration path-integral Monte Carlo (CPIMC), ${ }^{14}$ density matrix quantum Monte Carlo (DMQMC) ${ }^{15}$ with the initiator (iDMQMC) approximation, ${ }^{26}$ permutation-blocking path integral Monte Carlo (PB-PIMC), ${ }^{16}$ and restricted path integral Monte Carlo (RPIMC). ${ }^{12}$ The warm-dense UEG can be completely characterized by its density (or Wigner-Seitz radius, $r_{s}$ ) and its temperature, $\theta$, given in units of the Fermi energy. In general, CPIMC and DMQMC are expected to perform better at high density (low $r_{s}$ ), while PBPIMC and RPIMC are expected to be more reliable at low density (high $r_{s}$ ). Finite temperature QMC methods are usually more reliable at higher temperatures for which the sign problem is less severe. We do not report results for $r_{s}<0.5$ or for $\theta>1$ since a variety of methods including FT-CCSD should be reliable in these limits.

In the FT-CCSD calculations shown here, there are two sources of error: the finite basis set and the neglect of high-order excitations (triples, quadruples, etc.). Additionally, FT-CC results in the grand canonical ensemble will differ from QMC calculations in the canonical ensemble for a finite number of electrons. In other words, the finite-size error will be different in canonical and grand canonical ensembles. In Appendix D, we describe two methods of basis set extrapolation and comment on the magnitude of the basis set error in these calculations. In Figs. 5 and 6, we show the exchangecorrelation energy of the warm-dense polarized UEG as a function of $r_{s}$ as computed with FT-CCSD and a variety of QMC methods at reduced temperatures of 0.5 and 0.25 , respectively. For $r_{s} \leq 2$, FT-CCSD agrees well with CPIMC and iDMQMC, which should be reliable in this region (see Ref. 29, Sec. 5.7). For $r_{s}=4$, FTCCSD underestimates the magnitude of the exchange-correlation at both temperatures shown here. This is likely due to the neglect of triples. At zero temperature, the triples are estimated to account for $\sim 15 \%$ of the correlation energy at $r_{s}=4,{ }^{85}$ and this is consistent with what we see in the warm dense regime. We expect the finite-basis error to be significant, especially at $\theta=0.5$, and the good

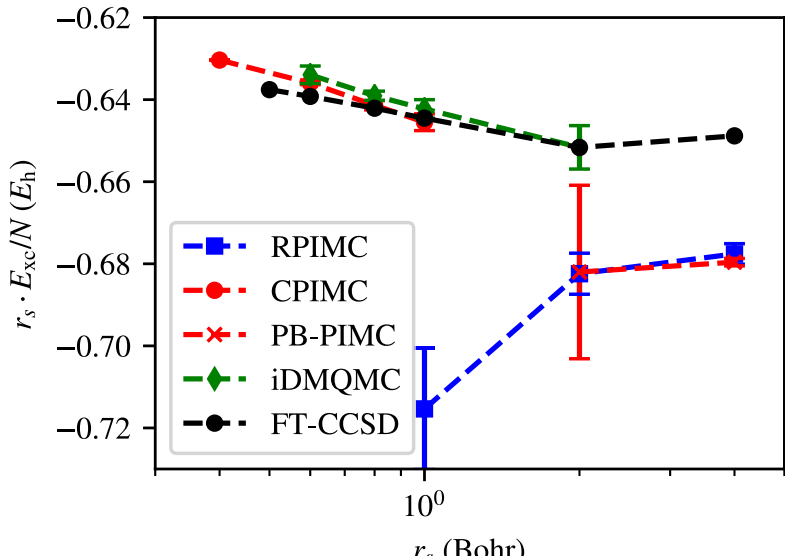

FIG. 5. The exchange-correlation energy of the $N=33$ polarized UEG as a function of $r_{s}$ for $\theta=0.5$. The exchange-correlation energy is scaled by $r_{s}$ to make the scale of the plot more uniform. The FT-CCSD calculations are performed in a basis of 123 plane-waves. The close agreement between FT-CC and QMC approaches in this basis set is likely due to a favorable cancellation of errors.

agreement at low $r_{s}$ for $\theta=0.5$ is likely due to a cancellation of errors.

In Figs. 7 and 8, we show the analogous calculations for the $N=66$ unpolarized UEG. For $N=66$, differences between grand canonical and canonical ensembles should be smaller, and we expect the primary source of error to be the basis set for $r_{s} \leq 2$ and the neglect of higher excitations at $r_{s}=4$. In Appendix $\mathrm{D}$, we provide an analysis of the finite basis error, which supports this claim.

More detailed calculations are necessary to make definitive statements about this system. These include calculations in larger basis sets, calculations that allow for an estimate of triples, and

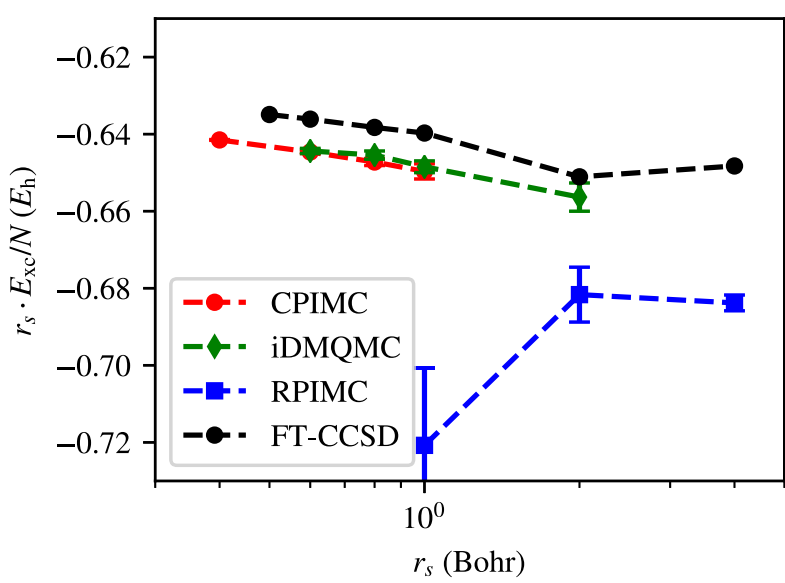

FIG. 6. The exchange-correlation energy of the $N=33$ polarized UEG as a function of $r_{s}$ for $\theta=0.25$. The exchange-correlation energy is scaled by $r_{s}$ to make the scale of the plot more uniform. The FT-CCSD calculations are extrapolated to the complete basis set limit using the E1 method described in Appendix D. For $r_{s}=4$, in particular, the neglect of triple excitations is likely the primary source of error. 


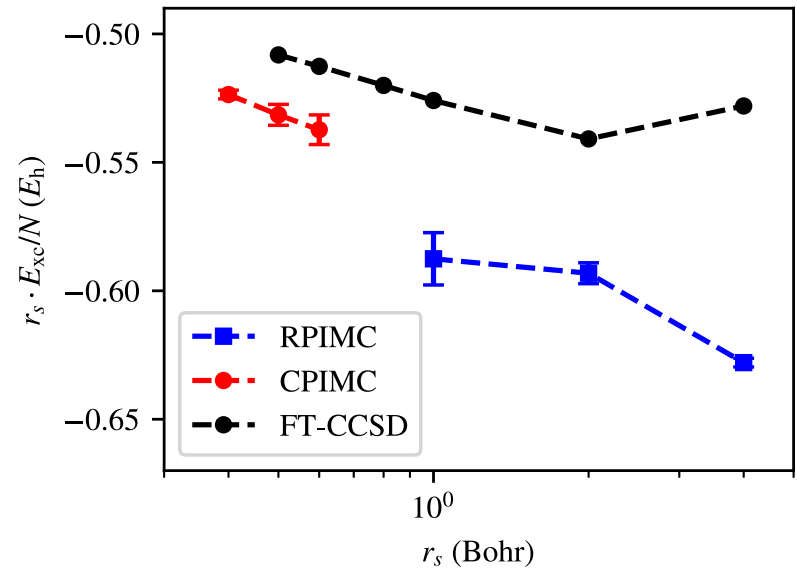

FIG. 7. The exchange-correlation energy of the $N=66$ unpolarized UEG as a function of $r_{s}$ for $\theta=0.5$. The exchange-correlation energy is scaled by $r_{s}$ to make the scale of the plot more uniform. The FT-CCSD calculations are performed in a basis of 123 plane-waves.

calculations that provide an estimate of the finite-size error. FTCC has the potential to provide systematically improvable results for the polarized and unpolarized UEGs for $r_{s} \leq 2$ and for a very wide range of temperatures. For $r_{s}=4$, some estimate of the triples contribution is probably necessary to obtain good results from CC theory. For even moderate $r_{s}$ (such as $r_{s}<4$ ), it is known that zero-temperature mean-field theory gives a wide range of broken symmetry solutions ${ }^{86}$ and the role of these broken symmetry states in subsequent coupled cluster calculations at finite temperature should be explored. Additionally, classifying correlation in terms of the order of the coupled cluster excitations can provide insight into the nature of correlation in this important system at finite temperature.

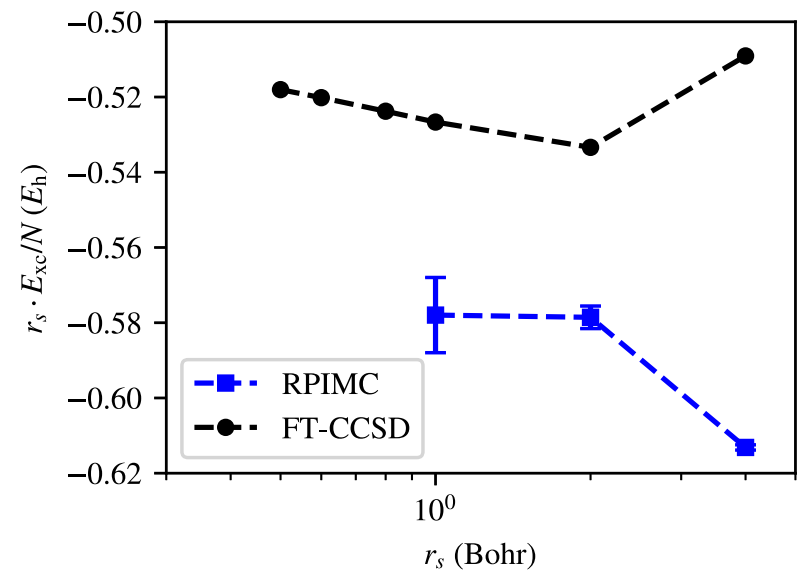

FIG. 8. The exchange-correlation energy of the $N=66$ unpolarized UEG as a function of $r_{s}$ for $\theta=0.25$. The exchange-correlation energy is scaled by $r_{s}$ to make the scale of the plot more uniform. The FT-CCSD calculations are performed in a basis of 123 plane-waves.

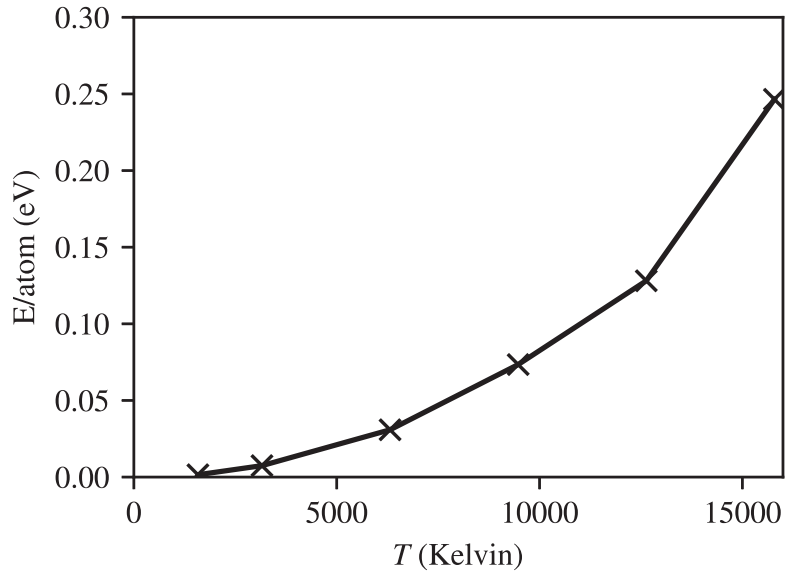

FIG. 9. The FT-CCSD energy per atom of diamond relative to the zero temperature CCSD energy in the same basis, $E_{\mathrm{FT}-\operatorname{CCSD}}(T)-E_{\mathrm{CCSD}}(0)$.

\section{Ab initio Hamiltonians}

Finally, we consider the application of FT-CCSD to the $a b$ initio problem. This problem is characterized by a number of difficulties, including

- converging to the thermodynamic limit in materials,

- larger one-particle basis sets and/or plane-wave cutoffs may be required at finite temperature because states with larger kinetic energy are populated,

- the large number of grid points required to control the numerical error at lower temperatures, and

- the inclusion of finite temperature nuclear effects

FT-CCSD in its current form is still too expensive for us to meaningfully address all these difficulties; however, we will nonetheless show that it is possible to apply FT-CCSD to the problem of $a b$ initio calculations on materials within the framework of local basis functions. In the following calculations, we use a minimal

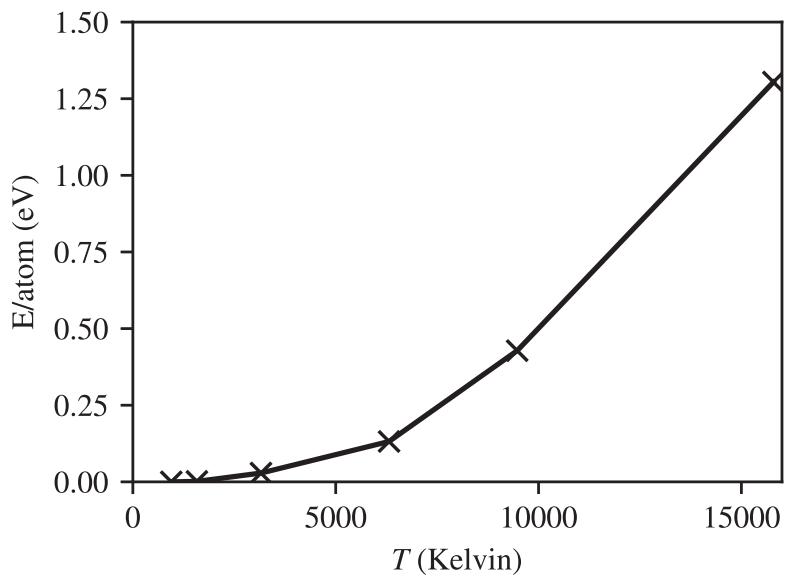

FIG. 10. The FT-CCSD energy per atom of silicon relative to the zero temperature CCSD energy in the same basis, $E_{\mathrm{FT}-\mathrm{CCSD}}(T)-E_{\mathrm{CCSD}}(0)$. 


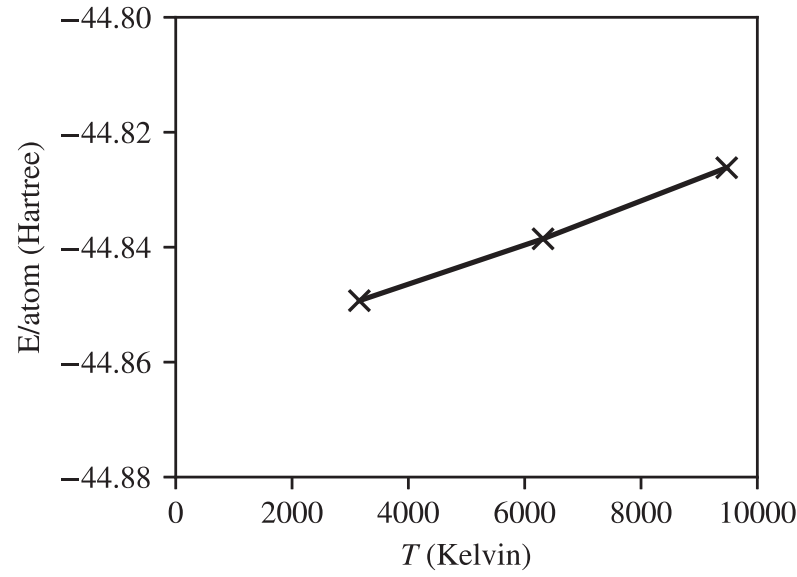

FIG. 11. The FT-CCSD energy $\left(E_{h}\right)$ per atom of copper. A finite-temperature Hartree-Fock reference with $T=0.01 E_{\mathrm{h}}$ was used for all points.

valence basis set of periodic Gaussian orbitals (SZV) ${ }^{87}$ and GTH pseudopotentials. ${ }^{88,89}$ The matrix elements have been obtained from the PySCF program package ${ }^{90}$ using plane-wave density fitting. ${ }^{91}$ Zero-temperature, ground-state CCSD calculations were performed as described in Ref. 92.

In Figs. 9 and 10, we show the energy per atom of diamond and silicon, respectively, relative to the zero temperature CCSD energy in the same basis set. In both of these calculations, a zero-temperature Hartree-Fock reference was used, and twist averaging ${ }^{93}$ over a $3 \times 3$ $\times 3 \mathrm{k}$-point grid at fixed, $\mu$ was used to partially alleviate finite-size errors. The twist average was computed by averaging the results of separate calculations centered at distinct k-points in the Brillouin zone. As the temperature approaches zero, the FT-CCSD energy approaches the zero-temperature, ground-state CCSD energy. The difference between the ground-state and finite-temperature energy is more pronounced for silicon relative to diamond because silicon has lower energy excited states.

Unfortunately, at lower temperatures, large orbital energy differences make integrating the FT-CCSD differential equations numerically unstable. This makes larger calculations difficult at lower temperatures. For example, for a two-atom supercell of copper metal twist averaged over $3 \times 3 \times 3$ mesh of k points, we were unable to reliably integrate the FT-CCSD equations much below $3000 \mathrm{~K}$ (see Fig. 11). Dealing with this difficulty is the subject of current investigations, but the initial results are nonetheless promising. It is rare to see finite-temperature calculations on materials where electron correlation is treated beyond the level of DFT, and FT-CCSD should be capable of providing valuable insight for such systems. Specifically, we are interested in transition metal oxides, such as vanadium oxide, where the electron correlation determines the thermal properties at low temperatures, and many metals where the electronic entropy determines the structural phase at low to moderate temperatures.

\section{CONCLUSIONS}

In this paper, we have discussed several aspects of FT-CCSD. All equations necessary for an efficient implementation have been presented, and some simple benchmarks have been provided to address the error incurred by restricting the orbital spaces and by numerical treatments of the imaginary time integration. Finally, we have shown the results for the 1D Hubbard model, the warm, dense UEG, and some simple $a b$ initio Hamiltonians. The 1D Hubbard model and the warm, dense UEG have allowed us to evaluate the strengths and weaknesses of FT-CCSD relative to exact or nearly exact results, and we find that, broadly speaking, FT-CCSD performs well for systems in which we might expect CCSD to perform well at zero temperature. For the warm, dense UEG, more calculations are needed to make truly definitive estimates of the exchangecorrelation energy, but FT-CCSD performs well over a wide range of temperatures and densities. Ab initio Hamiltonians present some difficulties because of the large system sizes necessary to approach the thermodynamic limit in materials applications and because of the relatively low temperatures necessary to obtain the results of relevance to many phenomena of interest. The results for small models of silicon and diamond clearly show that the ground state CCSD energy is the zero temperature limit of FT-CCSD, and we are currently pursuing solutions to the numerical problems at low temperatures.

Future work on finite-temperature coupled cluster methods is proceeding in three directions:

1. Technical improvements to address larger systems.

2. Theoretical improvements and approximations to more reliably treat lower temperatures.

3. Applications: more precise calculations on the UEG, benchmark $a b$ initio calculations on materials in the warm-dense regime, and $a b$ initio calculations of metallic systems at ambient temperatures.

\section{SUPPLEMENTARY MATERIAL}

See the supplementary material for tables of all the results shown in this work.

\section{ACKNOWLEDGMENTS}

This work was supported by the U.S. Department of Energy, Office of Science, via Grant No. SC0018140. The finite-temperature CC code relies on the PySCF software framework. The mean-field and periodic software infrastructure in PySCF has been developed with support from the U.S. National Science Foundation under Award No. 1657286. G.K.-L.C. was supported by the Simons Foundation via the Many-Electron Collaboration and via the Simons Investigator program. A.F.W. would like to thank Matthew Foulkes for helpful discussions and Chong Sun for help with the thermodynamic Bethe ansatz.

\section{APPENDIX A: FT-CCSD ENERGY, AMPLITUDE, and $\lambda$ EQUATIONS}

We will now state the FT-CCSD energy, amplitude, and $\lambda$ equations. We will use "thermal" one-electron and two-electron integrals,

$$
f_{i j} \equiv \sqrt{n_{i} n_{j}}\left[\langle i|f| j\rangle-\delta_{i j} \varepsilon_{i}\right]
$$




$$
\begin{aligned}
& f_{i a} \equiv \sqrt{n_{i}\left(1-n_{a}\right)}\langle i|f| a\rangle, \\
& f_{a i} \equiv \sqrt{n_{i}\left(1-n_{a}\right)}\langle a|f| i\rangle, \\
& f_{a b} \equiv \sqrt{\left(1-n_{a}\right)\left(1-n_{b}\right)}\left[\langle a|f| b\rangle-\delta_{a b} \varepsilon_{b}\right],
\end{aligned}
$$

$\langle i j|| a b\rangle \equiv \sqrt{n_{i} n_{j}\left(1-n_{a}\right)\left(1-n_{b}\right)}[\langle i j|V| a b\rangle-\langle i j|V| b a\rangle]$,

$\langle i j|| k a\rangle \equiv \sqrt{n_{i} n_{j} n_{k}\left(1-n_{a}\right)}[\langle i j|V| k a\rangle-\langle i j|V| a k\rangle] \quad$ etc.

The operator $f$ is the Fock operator of the finite-temperature meanfield density, the orbital energies, $\varepsilon_{p}$, define the mean field system, and $V$ is the two-particle Coulomb interaction.

The FT-CCSD grand potential is computed as

$$
\Omega_{C C}=\frac{1}{\beta} \sum_{y} g_{y} \mathrm{E}\left(\tau_{y}\right),
$$

where $g$ is the tensor of weights for some numerical integration scheme (see Sec. II C) and the kernel, E, is given by

$$
\mathrm{E}(\tau) \equiv \sum_{i a} f_{i a} s_{i}^{a}(\tau)+\frac{1}{4} \sum_{i j a b}\langle i j \| a b\rangle\left[s_{i j}^{a b}(\tau)+2 s_{i}^{a}(\tau) s_{j}^{b}(\tau)\right] .
$$

The FT-CCSD amplitude and $\lambda$ iterations can be written as

$$
\begin{gathered}
s_{i}^{a}\left(\tau_{y}\right)=-\tilde{S}_{i}^{a}\left(\tau_{y}\right), \\
s_{i j}^{a b}\left(\tau_{y}\right)=-\tilde{S}_{i j}^{a b}\left(\tau_{y}\right), \\
\lambda_{a}^{i}\left(\tau_{x}\right)=-\mathrm{L}_{a}^{i}\left(\tau_{x}\right), \\
\lambda_{a b}^{i j}\left(\tau_{x}\right)=-\mathrm{L}_{a b}^{i j}\left(\tau_{x}\right) .
\end{gathered}
$$

If the integral form of the equations is solved, we will use the following quadrature approximations to the integrated quantities:

$$
\begin{aligned}
& \tilde{\mathrm{S}}_{v}\left(\tau_{y}\right) \equiv \sum_{x} G_{x}^{y} e^{\Delta_{v}\left(\tau_{x}-\tau_{y}\right)} S_{v}\left(\tau_{x}\right), \\
& \tilde{\lambda}^{v}\left(\tau_{x}\right) \equiv \sum_{y} g_{y} \frac{G_{x}^{y}}{g_{x}} e^{\Delta_{v}\left(\tau_{x}-\tau_{y}\right)} \lambda^{v}\left(\tau_{y}\right) .
\end{aligned}
$$

If instead the differential form of the equations is propagated in imaginary time, the $s$ and $\tilde{\lambda}$ amplitudes are computed directly from the $S$ and L kernels. In either case, the utility of these definitions lies in the fact that the S and L kernels are local in time and are closely related to the ground state CCSD equations. For the singles, we find that

$$
\begin{aligned}
S_{i}^{a}\left(\tau_{x}\right)= & f_{a i}+\sum_{b} f_{a b} s_{i}^{b}\left(\tau_{x}\right)-\sum_{j} f_{j i} s_{j}^{a}\left(\tau_{x}\right)+\sum_{j b}\langle j a \| b i\rangle s_{j}^{b}\left(\tau_{x}\right)+\sum_{j b} f_{j b} s_{i j}^{a b}\left(\tau_{x}\right)+\frac{1}{2} \sum_{j b c}\langle a j \| b c\rangle s_{i j}^{b c}\left(\tau_{x}\right)-\frac{1}{2} \sum_{j k b}\langle j k \| i b\rangle s_{j k}^{a b}\left(\tau_{x}\right) \\
& -\sum_{j b} f_{j b} s_{i}^{b}\left(\tau_{x}\right) s_{j}^{a}\left(\tau_{x}\right)+\sum_{j b c}\langle j a \| b c\rangle s_{j}^{b}\left(\tau_{x}\right) s_{i}^{c}\left(\tau_{x}\right)-\sum_{j k b}\langle j k \| b i\rangle s_{j}^{b}\left(\tau_{x}\right) s_{k}^{a}\left(\tau_{x}\right)-\frac{1}{2} \sum_{j k b c}\langle j k \| b c\rangle s_{i}^{b}\left(\tau_{x}\right) s_{j k}^{a c}\left(\tau_{x}\right) \\
& -\frac{1}{2} \sum_{j k b c}\langle j k \| b c\rangle s_{j}^{a}\left(\tau_{x}\right) s_{i k}^{b c}\left(\tau_{x}\right)+\sum_{j k b c}\langle j k \| b c\rangle s_{j}^{b}\left(\tau_{x}\right) s_{k i}^{c a}\left(\tau_{x}\right)+\sum_{j k c d}\langle j k \| b c\rangle s_{i}^{b}\left(\tau_{x}\right) s_{j}^{c}\left(\tau_{x}\right) s_{k}^{a}\left(\tau_{x}\right)
\end{aligned}
$$

Similarly, for the doubles,

$$
\begin{aligned}
S_{i j}^{a b}\left(\tau_{x}\right)= & \langle a b \| i j\rangle+P(i j) \sum_{c}\langle a b \| c j\rangle s_{i}^{c}\left(\tau_{x}\right)-P(a b) \sum_{k}\langle k b \| i j\rangle s_{k}^{a}\left(\tau_{x}\right)+P(a b) \sum_{c} f_{b c} s_{i j}^{a c}\left(\tau_{x}\right)-P(i j) \sum_{k} f_{k j} s_{i k}^{a b}\left(\tau_{x}\right)+\frac{1}{2} \sum_{c d}\langle a b \| c d\rangle s_{i j}^{c d}\left(\tau_{x}\right) \\
& +\frac{1}{2} \sum_{k l}\langle k l \| i j\rangle s_{k l}^{a b}\left(\tau_{x}\right)+P(i j) P(a b) \sum_{k c}\langle k b \| c j\rangle s_{i k}^{a c}\left(\tau_{x}\right)+\frac{1}{2} P(i j) \sum_{c d}\langle a b \| c d\rangle s_{i}^{c}\left(\tau_{x}\right) s_{j}^{d}\left(\tau_{x}\right)+\frac{1}{2} P(a b) \sum_{k l}\langle k l \| i j\rangle s_{k}^{a}\left(\tau_{x}\right) s_{l}^{b}\left(\tau_{x}\right) \\
& -P(i j) P(a b) \sum_{k c}\langle a k \| c j\rangle s_{i}^{c}\left(\tau_{x}\right) s_{k}^{b}\left(\tau_{x}\right)-P(i j) \sum_{k c} f_{k c} s_{i}^{c}\left(\tau_{x}\right) s_{k j}^{a b}\left(\tau_{x}\right)-P(a b) \sum_{k c} f_{k c} s_{k}^{a}\left(\tau_{x}\right) s_{i j}^{c b}\left(\tau_{x}\right)+P(a b) \sum_{k c d}\langle k a \| c d\rangle s_{k}^{c}\left(\tau_{x}\right) s_{i j}^{d b}\left(\tau_{x}\right) \\
& -P(i j) \sum_{k l c}\langle k l \| c i\rangle s_{k}^{c}\left(\tau_{x}\right) s_{l j}^{a b}\left(\tau_{x}\right)+P(i j) P(a b) \sum_{k c d}\langle a k \| c d\rangle s_{i}^{c}\left(\tau_{x}\right) s_{k j}^{d b}\left(\tau_{x}\right)-P(i j) P(a b) \sum_{k l c}\langle k l \| i c\rangle s_{k}^{a}\left(\tau_{x}\right) s_{l j}^{c b}\left(\tau_{x}\right) \\
& +\frac{1}{2} P(i j) \sum_{k l c}\langle k l \| c j\rangle s_{i}^{c}\left(\tau_{x}\right) s_{k l}^{a b}\left(\tau_{x}\right)-\frac{1}{2} P(a b) \sum_{k c d}\langle k b \| c d\rangle s_{k}^{a}\left(\tau_{x}\right) s_{i j}^{c d}\left(\tau_{x}\right)+\frac{1}{4} \sum_{k l c d}\langle k l \| c d\rangle s_{i j}^{c d}\left(\tau_{x}\right) s_{k l}^{a b}\left(\tau_{x}\right) \\
& +\frac{1}{2} P(i j) P(a b) \sum_{k l c d}\langle k l \| c d\rangle s_{i k}^{a c}\left(\tau_{x}\right) s_{l j}^{d b}\left(\tau_{x}\right)-\frac{1}{2} P(a b) \sum_{k l c d}\langle k l \| c d\rangle s_{k l}^{c a}\left(\tau_{x}\right) s_{i j}^{d b}\left(\tau_{x}\right)-\frac{1}{2} P(i j) \sum_{k l c d}\langle k l \| c d\rangle s_{k i}^{c d}\left(\tau_{x}\right) s_{l j}^{a b}\left(\tau_{x}\right) \\
& -\frac{1}{2} P(i j) P(a b) \sum_{k c d}\langle k b \| c d\rangle s_{i}^{c}\left(\tau_{x}\right) s_{k}^{a}\left(\tau_{x}\right) s_{j}^{d}\left(\tau_{x}\right)+\frac{1}{2} P(i j) P(a b) \sum_{k l c}\langle k l \| c j\rangle s_{i}^{c}\left(\tau_{x}\right) s_{k}^{a}\left(\tau_{x}\right) s_{l}^{b}\left(\tau_{x}\right) \\
& +\frac{1}{4} P(i j) \sum_{k l c d}\langle k l \| c d\rangle s_{i}^{c}\left(\tau_{x}\right) s_{j}^{d}\left(\tau_{x}\right) s_{k l}^{a b}\left(\tau_{x}\right)+\frac{1}{4} P(a b) \sum_{k l c d}\langle k l \| c d\rangle s_{k}^{a}\left(\tau_{x}\right) s_{l}^{b}\left(\tau_{x}\right) s_{i j}^{c d}\left(\tau_{x}\right) \\
& -P(i j) P(a b) \sum_{k l c d}\langle k l \| c d\rangle s_{i}^{c}\left(\tau_{x}\right) s_{k}^{a}\left(\tau_{x}\right) s_{l j}^{d b}\left(\tau_{x}\right)-P(i j) \sum_{k l c d}\langle k l \| c d\rangle s_{k}^{c}\left(\tau_{x}\right) s_{i}^{d}\left(\tau_{x}\right) s_{l j}^{a b}\left(\tau_{x}\right) \\
& -P(a b) \sum_{k l c d}\langle k l \| c d\rangle s_{k}^{c}\left(\tau_{x}\right) s_{l}^{a}\left(\tau_{x}\right) s_{i j}^{d b}\left(\tau_{x}\right)+\frac{1}{4} P(i j) P(a b) \sum_{k l c d}\langle k l \| c d\rangle s_{i}^{c}\left(\tau_{x}\right) s_{k}^{a}\left(\tau_{x}\right) s_{l}^{b}\left(\tau_{x}\right) s_{j}^{d}\left(\tau_{x}\right) .
\end{aligned}
$$


The kernel $\mathrm{L}$ is also local in time and is equal to the $\operatorname{CCSD} \lambda$ equations evaluated with $\tilde{\lambda}$,

$$
\begin{aligned}
& \mathrm{L}_{a}^{i}\left(\tau_{x}\right)=f_{i a}+\sum_{b} \tilde{\lambda}_{b}^{i}\left(\tau_{x}\right) f_{b a}-\sum_{j} \tilde{\lambda}_{a}^{j}\left(\tau_{x}\right) f_{i j}+\sum_{j b} \tilde{\lambda}_{b}^{j}\left(\tau_{x}\right)\langle b i \| j a\rangle+\sum_{j b}\langle i j \| a b\rangle s_{j}^{b}\left(\tau_{x}\right)-\sum_{j b} \tilde{\lambda}_{a}^{j}\left(\tau_{x}\right) f_{i b} b s_{j}^{b}\left(\tau_{x}\right)-\sum_{j b} \tilde{\lambda}_{b}^{i}\left(\tau_{x}\right) f_{j a} s_{j}^{b}\left(\tau_{x}\right) \\
& +\sum_{j b c} \tilde{\lambda}_{c}^{i}\left(\tau_{x}\right)\langle c j \| a b\rangle s_{j}^{b}\left(\tau_{x}\right)-\sum_{j k b} \tilde{\lambda}_{a}^{k}\left(\tau_{x}\right)\langle i j \| k b\rangle s_{j}^{b}\left(\tau_{x}\right)+\sum_{j b c} \tilde{\lambda}_{c}^{j}\left(\tau_{x}\right)\langle c i \| b a\rangle s_{j}^{b}\left(\tau_{x}\right)-\sum_{j k b} \tilde{\lambda}_{b}^{k}\left(\tau_{x}\right)\langle j i \| k a\rangle s_{j}^{b}\left(\tau_{x}\right) \\
& -\frac{1}{2} \sum_{j k b c} \tilde{\lambda}_{a}^{j}\left(\tau_{x}\right)\langle i k \| b c\rangle s_{j k}^{b c}\left(\tau_{x}\right)-\frac{1}{2} \sum_{j k b c} \tilde{\lambda}_{b}^{i}\left(\tau_{x}\right)\langle j k \| \mid a c\rangle s_{j k}^{b c}\left(\tau_{x}\right)+\sum_{j k b c} \tilde{\lambda}_{b}^{j}\left(\tau_{x}\right)\langle k i \| c a\rangle s_{j k}^{b c}\left(\tau_{x}\right)-\sum_{j k b c} \tilde{\lambda}_{a}^{j}\left(\tau_{x}\right)\langle i k \| b c\rangle s_{j}^{b}\left(\tau_{x}\right) s_{k}^{c}\left(\tau_{x}\right) \\
& -\sum_{j k b c} \tilde{\lambda}_{b}^{i}\left(\tau_{x}\right)\langle j k \| a c\rangle s_{j}^{b}\left(\tau_{x}\right) s_{k}^{c}\left(\tau_{x}\right)-\sum_{j k b c} \tilde{\lambda}_{b}^{k}\left(\tau_{x}\right)\langle j i \| c a\rangle s_{j}^{b}\left(\tau_{x}\right) s_{k}^{c}\left(\tau_{x}\right)+\frac{1}{2} \sum_{j b c} \tilde{\lambda}_{c b}^{i j}\left(\tau_{x}\right)\langle c b \| a j\rangle-\frac{1}{2} \sum_{j k b} \tilde{\lambda}_{a b}^{k j}\left(\tau_{x}\right)\langle i b \| k j\rangle \\
& -\sum_{j k b c} \tilde{\lambda}_{a c}^{j k}\left(\tau_{x}\right)\langle i c \| b k\rangle s_{j}^{b}\left(\tau_{x}\right)-\sum_{j k b c} \tilde{\lambda}_{b c}^{i k}\left(\tau_{x}\right)\langle j c \| a k\rangle s_{j}^{b}\left(\tau_{x}\right)+\frac{1}{2} \sum_{j b c d} \tilde{\lambda}_{c d}^{i j}\left(\tau_{x}\right)\langle c d \| a b\rangle s_{j}^{b}\left(\tau_{x}\right)+\frac{1}{2} \sum_{j k l b} \tilde{\lambda}_{a b}^{k l}\left(\tau_{x}\right)\langle i j \| k l\rangle s_{j}^{b}\left(\tau_{x}\right) \\
& -\frac{1}{2} \sum_{j k b c} \tilde{\lambda}_{b a}^{j k}\left(\tau_{x}\right) f_{i c} s_{j k}^{b c}\left(\tau_{x}\right)-\frac{1}{2} \sum_{j k b c} \tilde{\lambda}_{b c}^{j i}\left(\tau_{x}\right) f_{k a} s_{j k}^{b c}\left(\tau_{x}\right)+\frac{1}{2} \sum_{j k b c d} \tilde{\lambda}_{b d}^{j k}\left(\tau_{x}\right)\langle d i \| c a\rangle s_{j k}^{b c}\left(\tau_{x}\right)-\frac{1}{2} \sum_{j k l b c} \tilde{\lambda}_{b c}^{j l}\left(\tau_{x}\right)\langle k i \| l a\rangle s_{j k}^{b c}\left(\tau_{x}\right) \\
& +\sum_{j k b c d} \tilde{\lambda}_{b d}^{j i}\left(\tau_{x}\right)\langle k d \| \mid c a\rangle s_{j k}^{b c}\left(\tau_{x}\right)-\sum_{j k l b c} \tilde{\lambda}_{b a}^{j l}\left(\tau_{x}\right)\langle k i \| c l\rangle s_{j k}^{b c}\left(\tau_{x}\right)-\frac{1}{4} \sum_{j k b c d} \tilde{\lambda}_{a d}^{j k}\left(\tau_{x}\right)\langle i d \| b c\rangle s_{j k}^{b c}\left(\tau_{x}\right)+\frac{1}{4} \sum_{j k l b c} \tilde{\lambda}_{b c}^{i l}\left(\tau_{x}\right)\langle j k \| a l\rangle s_{j k}^{b c}\left(\tau_{x}\right) \\
& -\sum_{j k b c d} \tilde{\lambda}_{d b}^{i k}\left(\tau_{x}\right)\langle d j \| a c\rangle s_{j}^{b}\left(\tau_{x}\right) s_{k}^{c}\left(\tau_{x}\right)+\sum_{j k l b c} \tilde{\lambda}_{a b}^{l k}\left(\tau_{x}\right)\langle i j \| l c\rangle s_{j}^{b}\left(\tau_{x}\right) s_{k}^{c}\left(\tau_{x}\right)-\frac{1}{2} \sum_{j k b c d} \tilde{\lambda}_{a d}^{j k}\left(\tau_{x}\right)\langle i d \| b c\rangle s_{j}^{b}\left(\tau_{x}\right) s_{k}^{c}\left(\tau_{x}\right) \\
& +\frac{1}{2} \sum_{j k l b c} \tilde{\lambda}_{b c}^{i l}\left(\tau_{x}\right)\langle j k \| a d\rangle s_{j}^{b}\left(\tau_{x}\right) s_{k}^{c}\left(\tau_{x}\right)-\frac{1}{2} \sum_{j k l b c d} \tilde{\lambda}_{c a}^{k l}\left(\tau_{x}\right)\langle i j \| d b\rangle s_{j}^{b}\left(\tau_{x}\right) s_{k l}^{c d}\left(\tau_{x}\right)-\frac{1}{2} \sum_{j k l b c d} \tilde{\lambda}_{c d}^{k i}\left(\tau_{x}\right)\langle l j \| a b\rangle s_{j}^{b}\left(\tau_{x}\right) s_{k l}^{c d}\left(\tau_{x}\right) \\
& -\sum_{j k l b c d} \tilde{\lambda}_{a d}^{j l}\left(\tau_{x}\right)\langle i k \| b c\rangle s_{j}^{b}\left(\tau_{x}\right) s_{k l}^{c d}\left(\tau_{x}\right)-\sum_{j k l b c d} \tilde{\lambda}_{b d}^{i l}\left(\tau_{x}\right)\langle j k \| \mid a c\rangle s_{j}^{b}\left(\tau_{x}\right) s_{k l}^{c d}\left(\tau_{x}\right)+\frac{1}{4} \sum_{j k l b c d} \tilde{\lambda}_{a b}^{k l}\left(\tau_{x}\right)\langle i j \| c d\rangle s_{j}^{b}\left(\tau_{x}\right) s_{k l}^{c d}\left(\tau_{x}\right) \\
& +\frac{1}{4} \sum_{j k l b c d} \tilde{\lambda}_{c d}^{i j}\left(\tau_{x}\right)\langle k l|| a b\rangle s_{j}^{b}\left(\tau_{x}\right) s_{k l}^{c d}\left(\tau_{x}\right)-\frac{1}{2} \sum_{j k l b c d} \tilde{\lambda}_{c b}^{k l}\left(\tau_{x}\right)\langle j i \| d a\rangle s_{j}^{b}\left(\tau_{x}\right) s_{k l}^{c d}\left(\tau_{x}\right)-\frac{1}{2} \sum_{j k l b c d} \tilde{\lambda}_{c d}^{k j}\left(\tau_{x}\right)\langle l i \| b a\rangle s_{j}^{b}\left(\tau_{x}\right) s_{k l}^{c d}\left(\tau_{x}\right) \\
& +\frac{1}{2} \sum_{j k l b c d} \tilde{\lambda}_{a c}^{j l}\left(\tau_{x}\right)\langle i k \| b d\rangle s_{j}^{b}\left(\tau_{x}\right) s_{k}^{c}\left(\tau_{x}\right) s_{l}^{d}\left(\tau_{x}\right)+\frac{1}{2} \sum_{j k l b c d} \tilde{\lambda}_{b c}^{i l}\left(\tau_{x}\right)\langle j k \| \mid a d\rangle s_{j}^{b}\left(\tau_{x}\right) s_{k}^{c}\left(\tau_{x}\right) s_{l}^{d}\left(\tau_{x}\right), \\
& \mathrm{L}_{a b}^{i j}\left(\tau_{x}\right)=\langle i j \| a b\rangle+P(i j) P(a b) f_{i a} \tilde{\lambda}_{b}^{j}\left(\tau_{x}\right)+P(i j) \sum_{c} \tilde{\lambda}_{c}^{i}\left(\tau_{x}\right)\langle c j \| a b\rangle-P(a b) \sum_{k} \tilde{\lambda}_{a}^{k}\left(\tau_{x}\right)\langle i j \| k b\rangle+P(i j) P(a b) \sum_{k c} \tilde{\lambda}_{b}^{j}\left(\tau_{x}\right)\langle i k \| a c\rangle s_{k}^{c}\left(\tau_{x}\right) \\
& -P(i j) \sum_{k c} \tilde{\lambda}_{c}^{i}\left(\tau_{x}\right)\langle k l \| a b\rangle s_{k}^{c}\left(\tau_{x}\right)-P(a b) \sum_{k c} \tilde{\lambda}_{a}^{k}\left(\tau_{x}\right)\langle i j \| c b\rangle s_{k}^{c}\left(\tau_{x}\right)+P(a b) \sum_{c} \tilde{\lambda}_{a c}^{i j}\left(\tau_{x}\right) f_{c b}-P(i j) \sum_{k} \tilde{\lambda}_{a b}^{i k}\left(\tau_{x}\right) f_{j k}+\frac{1}{2} \sum_{c d} \tilde{\lambda}_{c d}^{i j}\left(\tau_{x}\right)\langle c d \| a b\rangle \\
& +\frac{1}{2} \sum_{k l} \tilde{\lambda}_{a b}^{k l}\left(\tau_{x}\right)\langle i j \| k l\rangle+P(i j) P(a b) \sum_{k c} \tilde{\lambda}_{a c}^{i k}\left(\tau_{x}\right)\langle c j \| k b\rangle-P(i j) \sum_{k c} \tilde{\lambda}_{a b}^{i k}\left(\tau_{x}\right) f_{j c} s_{k}^{c}\left(\tau_{x}\right)-P(a b) \sum_{k c} \tilde{\lambda}_{a c}^{i j}\left(\tau_{x}\right) f_{k b} s_{k}^{c}\left(\tau_{x}\right) \\
& +P(a b) \sum_{k c d} \tilde{\lambda}_{a d}^{i j}\left(\tau_{x}\right)\langle d k \| b c\rangle s_{k}^{c}\left(\tau_{x}\right)-P(i j) \sum_{k l c} \tilde{\lambda}_{a b}^{i l}\left(\tau_{x}\right)\langle j k \| l c\rangle s_{k}^{c}\left(\tau_{x}\right)+P(i j) P(a b) \sum_{k c d} \tilde{\lambda}_{a d}^{i k}\left(\tau_{x}\right)\langle d j \| c b\rangle s_{k}^{c}\left(\tau_{x}\right) \\
& -P(i j) P(a b) \sum_{k l c} \tilde{\lambda}_{a c}^{i l}\left(\tau_{x}\right)\langle k j \| \mid l b\rangle s_{k}^{c}\left(\tau_{x}\right)-\sum_{k c d} \tilde{\lambda}_{c d}^{i j}\left(\tau_{x}\right)\langle k d \| a b\rangle s_{k}^{c}\left(\tau_{x}\right)+\sum_{k l c} \tilde{\lambda}_{a b}^{k l}\left(\tau_{x}\right)\langle i j \| c d\rangle s_{k}^{c}\left(\tau_{x}\right) \\
& \text { - P(ij) } \frac{1}{2} \sum_{k l c d} \tilde{\lambda}_{a b}^{i k}\left(\tau_{x}\right)\langle j l \| c d\rangle s_{k l}^{c d}\left(\tau_{x}\right)-P(a b) \frac{1}{2} \sum_{k l c d} \tilde{\lambda}_{a c}^{i j}\left(\tau_{x}\right)\langle k l \| b d\rangle s_{k l}^{c d}\left(\tau_{x}\right)+P(i j) P(a b) \sum_{k l c d} \tilde{\lambda}_{a c}^{i k}\left(\tau_{x}\right)\langle l j|| d b\rangle s_{k l}^{c d}\left(\tau_{x}\right) \\
& -P(a b) \frac{1}{2} \sum_{k l c d} \tilde{\lambda}_{c a}^{k l}\left(\tau_{x}\right)\langle i j \| d b\rangle s_{k l}^{c d}\left(\tau_{x}\right)-P(i j) \frac{1}{2} \sum_{k l c d} \tilde{\lambda}_{c d}^{k i}\left(\tau_{x}\right)\langle j \mid \| a b\rangle s_{k l}^{c d}\left(\tau_{x}\right)+\frac{1}{4} \sum_{k l c d} \tilde{\lambda}_{a b}^{k l}\left(\tau_{x}\right)\langle i j \| c d\rangle s_{k l}^{c d}\left(\tau_{x}\right) \\
& +\frac{1}{4} \sum_{k l c d} \tilde{\lambda}_{c d}^{i j}\left(\tau_{x}\right)\langle k l \| a b\rangle s_{k l}^{c d}\left(\tau_{x}\right)-P(i j) \sum_{k l c d} \tilde{\lambda}_{a b}^{i k}\left(\tau_{x}\right)\langle j l \| c d\rangle s_{k}^{c}\left(\tau_{x}\right) s_{l}^{d}\left(\tau_{x}\right)-P(a b) \sum_{k l c d} \tilde{\lambda}_{a c}^{i j}\left(\tau_{x}\right)\langle k l \| b d\rangle s_{k}^{c}\left(\tau_{x}\right) s_{l}^{d}\left(\tau_{x}\right) \\
& -\sum_{k l c d} \tilde{\lambda}_{a d}^{i k}\left(\tau_{x}\right)\langle i j \| c b\rangle s_{k}^{c}\left(\tau_{x}\right) s_{l}^{d}\left(\tau_{x}\right)+\frac{1}{2} \sum_{k l c d} \tilde{\lambda}_{a b}^{k l}\left(\tau_{x}\right)\langle i j \| c d\rangle s_{k}^{c}\left(\tau_{x}\right) s_{l}^{d}\left(\tau_{x}\right)+\frac{1}{2} \sum_{k l c d} \tilde{\lambda}_{c d}^{i j}\left(\tau_{x}\right)\langle k l|| a b\rangle s_{k}^{c}\left(\tau_{x}\right) s_{l}^{d}\left(\tau_{x}\right) .
\end{aligned}
$$


In practice, we use the intermediate scheme of Stanton and Gauss ${ }^{95,96}$ to compute S and L efficiently.

\section{APPENDIX B: FT-CCSD RESPONSE DENSITIES}

As we discussed in Sec. II D, the computation of derivatives can be efficiently implemented by computing response densities that can be contracted with the basis representation of operators to compute properties. In Sec. II D, we described a total of six terms relevant to the computation of derivatives. We will now describe how the contribution of all terms can be efficiently computed for FT-CCSD.

Terms of type 1 can be computed by evaluating the Lagrangian with the quantities,

$$
\frac{\partial E}{\partial \alpha}, \quad \frac{\partial S_{v}}{\partial \alpha},
$$

but it is more efficient to first form unrelaxed, normal-ordered 1- or 2-particle response densities, $\gamma_{N}$ or $\Gamma_{N}$, and then trace them with the appropriate operators when more than one property is desired. The expressions for these quantities are given in Eqs. (B2)-(B14) with implied summations.

The unrelaxed 1-RDM:

$$
\begin{aligned}
\frac{\left(\gamma_{N}\right)_{i a}}{\sqrt{n_{i}\left(1-n_{a}\right)}} & =g_{y} \tilde{\lambda}_{a}^{i}\left(\tau_{y}\right), \\
\frac{\left(\gamma_{N}\right)_{b a}}{\sqrt{\left(1-n_{b}\right)\left(1-n_{a}\right)}} & =g_{y} \tilde{\lambda}_{a}^{i}\left(\tau_{y}\right) s_{i}^{b}\left(\tau_{y}\right)+\frac{1}{2} g_{y} \tilde{\lambda}_{c b}^{k i}\left(\tau_{y}\right) s_{k i}^{c a}\left(\tau_{y}\right), \\
\frac{\left(\gamma_{N}\right)_{j i}}{\sqrt{n_{i} n_{j}}} & =-g_{y} \tilde{\lambda}_{a}^{j}\left(\tau_{y}\right) s_{i}^{a}\left(\tau_{y}\right)-\frac{1}{2} g_{y} \tilde{\lambda}_{c a}^{k j}\left(\tau_{y}\right) s_{k i}^{c a}\left(\tau_{y}\right),
\end{aligned}
$$

$$
\begin{aligned}
\frac{\left(\gamma_{N}\right)_{a i}}{\sqrt{n_{i}\left(1-n_{a}\right)}=} & g_{y} s_{i}^{a}\left(\tau_{y}\right)+g_{y} \tilde{\lambda}_{b}^{j}\left(\tau_{y}\right) s_{j i}^{b a}\left(\tau_{y}\right)-g_{y} \tilde{\lambda}_{b}^{j}\left(\tau_{y}\right) s_{i}^{b}\left(\tau_{y}\right) s_{j}^{a}\left(\tau_{y}\right) \\
& -\frac{1}{2} g_{y} \tilde{\lambda}_{b c}^{j k}\left(\tau_{y}\right) s_{i}^{b}\left(\tau_{y}\right) s_{j k}^{a c}\left(\tau_{y}\right) \\
& -\frac{1}{2} g_{y} \tilde{\lambda}_{b c}^{j k}\left(\tau_{y}\right) s_{j}^{a}\left(\tau_{y}\right) s_{i k}^{b c}\left(\tau_{y}\right) .
\end{aligned}
$$

The unrelaxed 2-RDM:

$$
\begin{gathered}
\frac{\left(\Gamma_{N}\right)_{i j a b}}{\sqrt{n_{j} n_{j}\left(1-n_{a}\right)\left(1-n_{b}\right)}}=g_{y} \tilde{\lambda}_{a b}^{i j}\left(\tau_{y}\right), \\
\frac{\left(\Gamma_{N}\right)_{c i a b}}{\sqrt{\left(1-n_{c}\right) n_{i}\left(1-n_{a}\right)\left(1-n_{b}\right)}}=g_{y} \tilde{\lambda}_{a b}^{j i}\left(\tau_{y}\right) s_{j}^{c}\left(\tau_{y}\right),
\end{gathered}
$$

$$
\frac{\left(\Gamma_{N}\right)_{j k a i}}{\sqrt{n_{j} n_{k}\left(1-n_{a}\right) n_{i}}}=-g_{y} \tilde{\lambda}_{a b}^{j k}\left(\tau_{y}\right) s_{i}^{b}\left(\tau_{y}\right)
$$

$$
\begin{gathered}
\frac{\left(\Gamma_{N}\right)_{c d a b}}{\sqrt{\left(1-n_{c}\right)\left(1-n_{d}\right)\left(1-n_{a}\right)\left(1-n_{b}\right)}} \\
=+\frac{1}{2} g_{y} \tilde{\lambda}_{a b}^{k l}\left(\tau_{y}\right) s_{k l}^{c d}\left(\tau_{y}\right) \\
+P(c d) g_{y} \frac{1}{2} \tilde{\lambda}_{c d}^{k l}\left(\tau_{y}\right) s_{k}^{c}\left(\tau_{y}\right) s_{l}^{d}\left(\tau_{y}\right), \\
\frac{\left(\Gamma_{N}\right)_{b j i a}}{\sqrt{\left(1-n_{b}\right) n_{j} n_{i}\left(1-n_{a}\right)}}=g_{y} \tilde{\lambda}_{a}^{j}\left(\tau_{y}\right) s_{i}^{b}\left(\tau_{y}\right)+g_{y} \tilde{\lambda}_{c a}^{k j}\left(\tau_{y}\right) s_{k i}^{c b}\left(\tau_{y}\right) \\
-g_{y} \tilde{\lambda}_{a c}^{k j}\left(\tau_{y}\right) s_{k}^{b}\left(\tau_{y}\right) s_{i}^{c}\left(\tau_{y}\right),
\end{gathered}
$$

$$
\frac{\left(\Gamma_{N}\right)_{k l i j}}{\sqrt{n_{k} n_{l} n_{i} n_{j}}}=\frac{1}{2} g_{y} \tilde{\lambda}_{c d}^{k l}\left(\tau_{y}\right) s_{i j}^{c d}\left(\tau_{y}\right)+P(i j) \frac{1}{2} g_{y} \tilde{\lambda}_{c d}^{k l}\left(\tau_{y}\right) s_{i}^{c}\left(\tau_{y}\right) s_{j}^{d}\left(\tau_{y}\right)
$$

$$
\begin{aligned}
& \frac{\left(\Gamma_{N}\right)_{b c a i}}{\sqrt{\left(1-n_{b}\right)(1-n)\left(1-n_{a}\right) n_{i}}}=g_{y} \tilde{\lambda}_{a}^{j}\left(\tau_{y}\right) s_{j i}^{b c}\left(\tau_{y}\right)+P(b c) g_{y} \tilde{\lambda}_{a}^{j}\left(\tau_{y}\right) s_{j}^{b}\left(\tau_{y}\right) s_{i}^{c}\left(\tau_{y}\right)+P(b c) \frac{1}{2} g_{y} \tilde{\lambda}_{d a}^{l k}\left(\tau_{y}\right) s_{l k}^{d b}\left(\tau_{y}\right) s_{i}^{c}\left(\tau_{y}\right) \\
& +P(b c) g_{y} \tilde{\lambda}_{a d}^{k l}\left(\tau_{y}\right) s_{i l}^{c d}\left(\tau_{y}\right) s_{k}^{b}\left(\tau_{y}\right)-\frac{1}{2} g_{y} \tilde{\lambda}_{a d}^{k l}\left(\tau_{y}\right) s_{k l}^{b c}\left(\tau_{y}\right) s_{i}^{d}\left(\tau_{y}\right)-g_{y} \tilde{\lambda}_{a d}^{k l}\left(\tau_{y}\right) s_{k}^{b}\left(\tau_{y}\right) s_{i}^{d}\left(\tau_{y}\right) s_{l}^{c}\left(\tau_{y}\right), \\
& \frac{\left(\Gamma_{N}\right)_{k a i j}}{\sqrt{n_{k}\left(1-n_{a}\right) n_{i} n_{j}}}=-g_{y} \tilde{\lambda}_{b}^{k}\left(\tau_{y}\right) s_{i j}^{b a}\left(\tau_{y}\right)-P(i j) g_{y} \tilde{\lambda}_{b}^{k}\left(\tau_{y}\right) s_{i}^{b}\left(\tau_{y}\right) s_{j}^{a}\left(\tau_{y}\right)-P(i j) \frac{1}{2} g_{y} \tilde{\lambda}_{b d}^{k l}\left(\tau_{y}\right) s_{i l}^{b d}\left(\tau_{y}\right) s_{j}^{a}\left(\tau_{y}\right) \\
& -P(i j) g_{y} \tilde{\lambda}_{b d}^{k l}\left(\tau_{y}\right) s_{j l}^{a d}\left(\tau_{y}\right) s_{i}^{b}\left(\tau_{y}\right)+\frac{1}{2} g_{y} \tilde{\lambda}_{d b}^{l k}\left(\tau_{y}\right) s_{j i}^{d b}\left(\tau_{y}\right) s_{l}^{a}\left(\tau_{y}\right)+g_{y} \tilde{\lambda}_{d b}^{l k}\left(\tau_{y}\right) s_{j}^{d}\left(\tau_{y}\right) s_{l}^{a}\left(\tau_{y}\right) s_{j}^{d}\left(\tau_{y}\right), \\
& \frac{\left(\Gamma_{N}\right)_{a b i j}}{\sqrt{\left(1-n_{a}\right)\left(1-n_{b}\right) n_{i} n_{j}}}=g_{y} s_{i j}^{a b}\left(\tau_{y}\right)+\frac{1}{2} P(i j, a b) g_{y} s_{i}^{a}\left(\tau_{y}\right) s_{j}^{b}\left(\tau_{y}\right)-P(a b) g_{y} \tilde{\lambda}_{c}^{k}\left(\tau_{y}\right) s_{i j}^{c b}\left(\tau_{y}\right) s_{k}^{a}\left(\tau_{y}\right)-P(i j) g_{y} \tilde{\lambda}_{c}^{k}\left(\tau_{y}\right) s_{k j}^{a b}\left(\tau_{y}\right) s_{i}^{c}\left(\tau_{y}\right) \\
& +P(i j, a b) g_{y} \tilde{\lambda}_{c}^{k}\left(\tau_{y}\right) s_{j k}^{b c}\left(\tau_{y}\right) s_{i}^{a}\left(\tau_{y}\right)-P(i j, a b) g_{y} \tilde{\lambda}_{c}^{k}\left(\tau_{y}\right) s_{k}^{a}\left(\tau_{y}\right) s_{i}^{c}\left(\tau_{y}\right) s_{j}^{b}\left(\tau_{y}\right)+\frac{1}{4} g_{y} \tilde{\lambda}_{c d}^{k l}\left(\tau_{y}\right) s_{k l}^{a b}\left(\tau_{y}\right) s_{i j}^{c d}\left(\tau_{y}\right) \\
& +\frac{1}{2} P(i j, a b) g_{y} \tilde{\lambda}_{c d}^{k l}\left(\tau_{y}\right) s_{k i}^{c a}\left(\tau_{y}\right) s_{l j}^{d b}\left(\tau_{y}\right)-\frac{1}{2} P(a b) g_{y} \tilde{\lambda}_{c d}^{k l}\left(\tau_{y}\right) s_{i j}^{a c}\left(\tau_{y}\right) s_{k l}^{b d}\left(\tau_{y}\right)-\frac{1}{2} P(i j) g_{y} \tilde{\lambda}_{c d}^{k l}\left(\tau_{y}\right) s_{i j}^{a b}\left(\tau_{y}\right) s_{j l}^{c d}\left(\tau_{y}\right) \\
& +\frac{1}{4} P(a b) g_{y} \tilde{\lambda}_{c d}^{k l}\left(\tau_{y}\right) s_{i j}^{c d}\left(\tau_{y}\right) s_{k}^{a}\left(\tau_{y}\right) s_{l}^{b}\left(\tau_{y}\right)+\frac{1}{4} P(i j) g_{y} \tilde{\lambda}_{c d}^{k l}\left(\tau_{y}\right) s_{k l}^{a b}\left(\tau_{y}\right) s_{i}^{c}\left(\tau_{y}\right) s_{j}^{d}\left(\tau_{y}\right) \\
& \text { - P(ij,ab)gy } \tilde{\lambda}_{c d}^{k l}\left(\tau_{y}\right) s_{j l}^{b d}\left(\tau_{y}\right) s_{k}^{a}\left(\tau_{y}\right) s_{i}^{c}\left(\tau_{y}\right)-\frac{1}{2} P(i j, a b) g_{y} \tilde{\lambda}_{c d}^{k l}\left(\tau_{y}\right) s_{j l}^{c d}\left(\tau_{y}\right) s_{k}^{b}\left(\tau_{y}\right) s_{i}^{a}\left(\tau_{y}\right) \\
& -\frac{1}{2} P(i j, a b) g_{y} \tilde{\lambda}_{c d}^{k l}\left(\tau_{y}\right) s_{k l}^{b d}\left(\tau_{y}\right) s_{j}^{c}\left(\tau_{y}\right) s_{i}^{a}\left(\tau_{y}\right)+\frac{1}{4} P(i j, a b) g_{y} \tilde{\lambda}_{c d}^{k l}\left(\tau_{y}\right) s_{k}^{a}\left(\tau_{y}\right) s_{i}^{c}\left(\tau_{y}\right) s_{l}^{b}\left(\tau_{y}\right) s d_{j}^{d}\left(\tau_{y}\right) .
\end{aligned}
$$


Recall that the indices $i$ and $a$ do not refer to disjoint subspaces, and therefore, the full unrelaxed density matrix can be written in the MO basis as

$$
\begin{aligned}
\left(\gamma_{N}\right)_{p q}= & \sum_{i a}\left(\gamma_{N}\right)_{i a} \delta_{i p} \delta_{a q}+\sum_{b a}\left(\gamma_{N}\right)_{b a} \delta_{b p} \delta_{a q} \\
& +\sum_{i j}\left(\gamma_{N}\right)_{j i} \delta_{j p} \delta_{i q}+\sum_{a i}\left(\gamma_{N}\right)_{a i} \delta_{a p} \delta_{i q} .
\end{aligned}
$$

We may compute the average of some operator, $X$, as

$$
\langle X\rangle=\sum_{p q}\left(\gamma_{N}\right)_{q p} X_{p q}^{(1)}+\sum_{p q} p_{q p} X_{p q}
$$

where $p$ is the mean-field 1-RDM,

$$
p_{q p}=\delta_{q p} n_{p}
$$

The expression for the average of 2-particle properties is analogous. The normal ordered $2-\mathrm{RDM}$ is given by

$$
\left(\Gamma_{N}\right)_{p q r s}=\left(\Gamma_{N}\right)_{i j a b} \delta_{i p} \delta_{j q} \delta_{r a} \delta_{s b}+\cdots
$$

The full unrelaxed 2-RDM additionally includes the contribution from the reference density,

$$
\begin{aligned}
\Gamma_{p q r s}= & \left(\Gamma_{N}\right)_{p q r s}+\frac{1}{2}\left[\left(\gamma_{N}\right)_{p r} p_{q s}-\left(\gamma_{N}\right)_{p s} p_{r q}\right] \\
& +\frac{1}{2}\left[p_{p r}\left(\gamma_{N}\right)_{q s}-p_{p s}\left(\gamma_{N}\right)_{r q}\right]+p_{p r} p_{q s}-p_{p s} p_{q r} .
\end{aligned}
$$

A two-electron observable, $Y$, can then be approximated by tracing its operator representation with the unrelaxed 2-RDM,

$$
\langle Y\rangle=\frac{1}{4} \sum_{p q r s} \Gamma_{p q r s} Y_{r s p q}
$$

It is also possible to partially relax the properties by including the response of the orbital energies and the occupation numbers. This involves three contributions:

$$
\frac{\partial \Omega^{(1)}}{\partial n_{i}} \frac{\partial n_{i}}{\partial \varepsilon_{i}} \frac{\partial \varepsilon_{i}}{\partial \alpha}, \quad \frac{\partial \mathcal{L}}{\partial n_{i}} \frac{\partial n_{i}}{\partial \varepsilon_{i}} \frac{\partial \varepsilon_{i}}{\partial \alpha}, \quad \frac{\partial \mathcal{L}}{\partial \varepsilon_{i}} \frac{\partial \varepsilon_{i}}{\partial \alpha} .
$$

Since the derivative of the orbital energies is just equal to

$$
\frac{\partial \varepsilon_{i}}{\partial \alpha}=\langle i|X| i\rangle
$$

for a 1-electron operator, the contribution due to the relaxation of the orbital energies and occupation numbers can be computed as

$$
\sum_{q} d_{q} X_{q}^{(0)}
$$

where

$$
d_{q}=\frac{\partial \Omega^{(1)}}{\partial n_{q}} \frac{\partial n_{q}}{\partial \varepsilon_{q}}+\frac{\partial \mathcal{L}}{\partial n_{q}} \frac{\partial n_{q}}{\partial \varepsilon_{q}}+\frac{\partial \mathcal{L}}{\partial \varepsilon_{q}} .
$$

The first term is most easily computed from the derivative of the first order correction to $\Omega$,

$$
\begin{aligned}
\frac{\partial \Omega^{(1)}}{\partial n_{q}} & =\left(h_{q q}-\varepsilon_{q}\right)+\sum_{j}[\langle q j|V| q j\rangle-\langle q j|V| j q\rangle] n_{j} \\
& =\langle q|f| q\rangle-\varepsilon_{q} .
\end{aligned}
$$

This contribution will be zero for a thermal Hartree-Fock reference.

The second term is most efficiently computed by first forming derivative integrals,

$$
\begin{gathered}
f_{a b}^{(q)}=\frac{\partial n_{q}}{\partial \varepsilon_{q}} \frac{\partial}{\partial n_{q}} f_{a b}, \\
\langle a b \| c d\rangle^{(q)}=\frac{\partial n_{q}}{\partial \varepsilon_{q}} \frac{\partial}{\partial n_{q}}\langle a b \| c d\rangle,
\end{gathered}
$$

and then contracting them with the unrelaxed, normal-ordered, 1and 2-RDMs,

$$
\left(\gamma_{N}\right)_{b a} f_{a b}^{(q)}+\left(\Gamma_{N}\right)_{c d a b}\langle a b \| c d\rangle^{(q)}+\cdots
$$

Note that while the derivatives of the Fock matrix are dense 3-index quantities because the Fock matrix involves sums over occupation numbers, the derivatives of the two-electron interaction are still only 4-index quantities because

$$
\begin{aligned}
\langle a b \| c d\rangle^{(q)}= & \langle a b \| c d\rangle^{(a)} \delta_{q a}+\langle a b \| c d\rangle^{(b)} \delta_{q b} \\
& +\langle a b \| c d\rangle^{(c)} \delta_{q c}+\langle a b \| c d\rangle^{(d)} \delta_{q d} .
\end{aligned}
$$

The remaining contribution is computed by taking the derivative of orbital energies that appear directly in the Lagrangian in the exponential factor. If the integral form of the equations is used, then this term can be computed directly,

$$
\frac{\partial \mathcal{L}}{\partial \varepsilon_{q}}=-\frac{1}{\beta} \sum_{y} g_{y} \lambda^{v}\left(\tau_{y}\right) \sum_{x}\left(\tau_{y}-\tau_{x}\right) G_{x}^{y} e^{\Delta_{v}\left(\tau_{x}-\tau_{y}\right)} \mathrm{S}_{v}\left(\tau_{x}\right) \frac{\partial \Delta_{v}}{\partial \varepsilon_{q}} .
$$

The label $v$ runs over all singles and doubles, and the derivatives of the energies differences are sparse in that

$$
\frac{\partial \Delta_{i}^{a}}{\partial \varepsilon_{q}}=\delta_{a q}-\delta_{i q}
$$

In the case that the differential form of the equations is used, this term must be calculated as

$$
\frac{1}{\beta} \int_{0}^{\beta} d \tau \mathrm{S}_{v}[\mathbf{s}(\tau)] \frac{\partial \tilde{\lambda}^{v}(\tau)}{\partial \varepsilon_{p}},
$$

where the derivative appearing under the integral can be propagated along with $\tilde{\lambda}$,

$$
\frac{d}{d \tau} \frac{\partial \tilde{\lambda}^{v}(\tau)}{\partial \varepsilon_{p}}=\Delta_{v} \frac{\partial \tilde{\lambda}^{v}(\tau)}{\partial \varepsilon_{p}}+\tilde{\lambda}(\tau) .
$$

Usually, $X^{(0)}$ contains all the diagonal elements and $X^{(1)}$ contains all the off-diagonal elements. In this case, we can construct one partially relaxed FT-CCSD density matrix as

$$
p_{q p}^{c c}=\left(\gamma_{N}\right)_{q p}\left(1-\delta_{q p}\right)+\delta_{q p}\left(d_{q}+n_{q}\right) .
$$

This is the FT-CCSD "density matrix," which incorporates the relaxation of the orbital energies and occupation numbers. 
We will not explicitly discuss the procedure for including orbital response (term 3), as we do not consider these terms in this work. However, the computation of the FT-CC Z-vector parallels closely the ground-state case, which is discussed in Ref. 97.

For the derivatives with respect to $\beta$, there are three additional terms that we must consider. Term 4 is just

$$
\text { (4) }=-\frac{1}{\beta} \Omega_{c c} \text {. }
$$

Term 5 can be computed specifically for a particular discretization by evaluating the Lagrangian with

$$
\frac{\partial G}{\partial \beta} \text { and } \frac{\partial g}{\partial \beta},
$$

respectively, or these terms can be computed as the derivative of the integration limits in the Lagrangian. This amounts to evaluating the integrand of $\mathcal{L}$ at $\tau=\beta$, and two methods will agree in the limit of a dense grid. Terms of type 6 are simple to write down due to the fact that the positions of the grid points depend linearly on $\beta$,

$$
\frac{\partial \tau_{y}}{\partial \beta}=\frac{\tau_{y}}{\beta},
$$

and therefore,

$$
\text { (6) }=-\frac{1}{\beta^{2}} \sum_{y} g_{y} \lambda^{v}\left(\tau_{y}\right) \sum_{x} \Delta_{v}\left(\tau_{y}-\tau_{x}\right) G_{x}^{y} e^{\Delta_{v}\left(\tau_{x}-\tau_{y}\right)} S_{v}\left(\tau_{x}\right) .
$$

This final term will vanish in the limit of a dense grid and can therefore be ignored without affecting the properties in the limit as $n_{g} \rightarrow \infty$.

\section{APPENDIX C: ENTROPY OF THE 1D HUBBARD MODEL}

In Fig. 12, the entropy of the 1D Hubbard model is plotted in more detail. FT-CCSD consistently underestimates the entropy at low temperatures, and this effect is more pronounced at for larger $U$.

\section{APPENDIX D: BASIS SET ERROR IN THE WARM, DENSE UEG}

The exchange-correlation energy is defined for a fixed number of electrons. In the grand canonical ensemble, we compute $E_{x c}$ for $N$ electrons in $M$ plane wave orbitals as

$$
E_{x c}(N, M)=E_{C C}\left(\mu_{C C}(M), M\right)+E_{0}\left(\mu_{C C}(M), M\right)-E_{0}\left(\mu_{0}(M), M\right),
$$

where $E_{C C}$ is the FT-CCSD exchange-correlation energy and $\mu_{C C}$ and $\mu_{0}$ are chosen separately so that each of the coupled cluster and reference systems has $N$ electrons. The simplest basis extrapolation technique, which we will refer to as "E1," extrapolates this quantity assuming that the basis dependence behaves asymptotically like $1 / M$. However, at higher temperatures, there will be significant finite-basis error in the computation of $E_{0}$, and we could also compute

$$
E_{x c}^{\prime}(N, M)=E_{C C}\left(\mu_{C C}(M), M\right)+E_{0}\left(\mu_{C C}(M), \infty\right)-E_{0}\left(\mu_{0}(M), \infty\right) .
$$
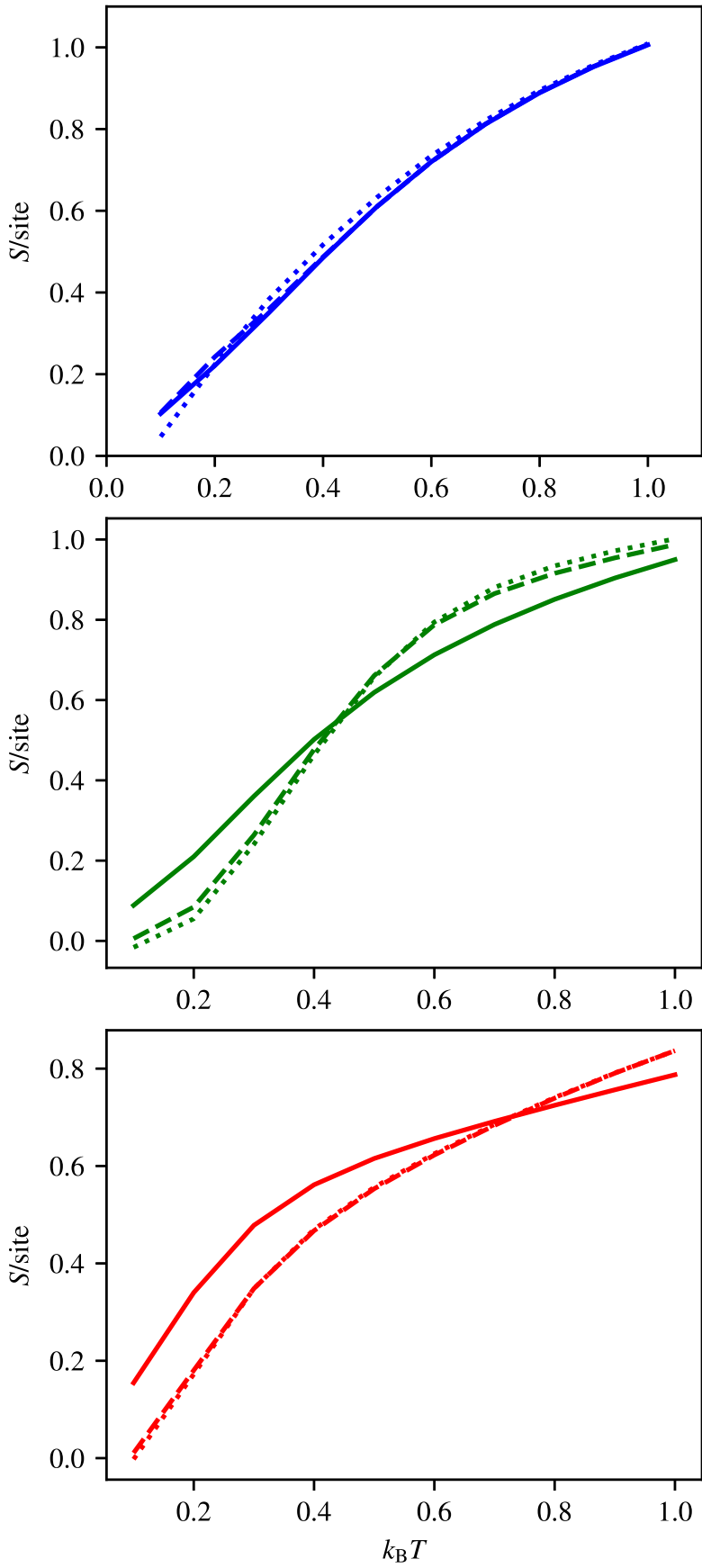

FIG. 12. More detailed view of the entropy per site of the 1D Hubbard model at hal filling for $U=2$ (blue, top), $U=4$ (green, middle), and $U=8$ (red, bottom). This is the same as row 3 (first column) of Fig. 3 .

The extrapolation of this quantity based on asymptotic $1 / M$ dependence, will be referred to as "E2." Other types of extrapolations are possible, but these two are sufficient for our purposes. 


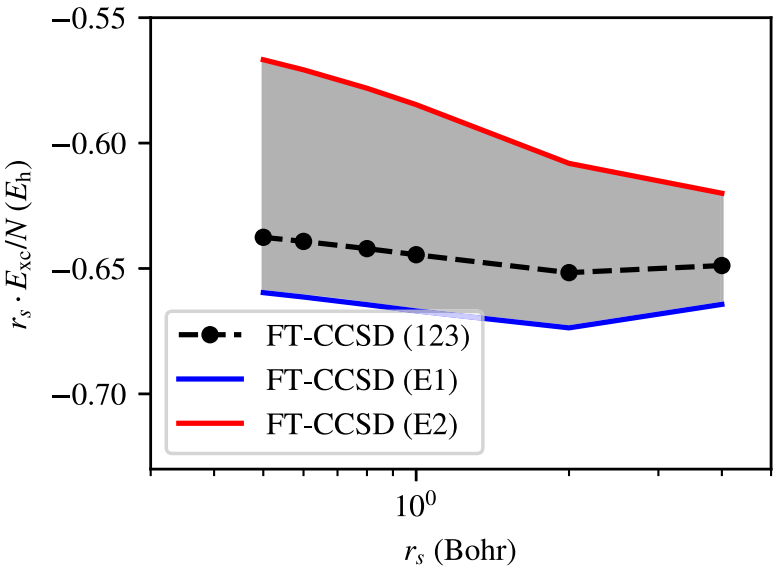

FIG. 13. The FT-CCSD exchange-correlation energy of the $N=33$ polarized UEG at a reduced temperature of $\theta=0.5$. The exchange-correlation energy is scaled by $r_{s}$ to make the scale of the plot more uniform. The solid lines are the extrapolated values based on the E1 (blue) and E2 (red) methods. The shaded region provides a rough estimate of the uncertainty in these extrapolations.

In Figs. 13 and 14, we plot the FT-CCSD exchange-correlation energy of the polarized UEG in a basis set of 123 plane waves. Additionally, we have extrapolated to the complete basis set limit with the E1 and E2 methods using basis set sizes of 93 and 123 plane waves. The difference in the two extrapolations, which should provide the same answer asymptotically, allows us to estimate the uncertainty in the basis set extrapolation. For $\theta=0.5$, the uncertainty is quite large, and there is no reason to think that either extrapolation is more reliable than the $M=123$ results. On the other hand, for $\theta=0.25$, both E1 and E2 methods provide similar results, which suggests that either may provide a better estimate than the $M=123$ results.

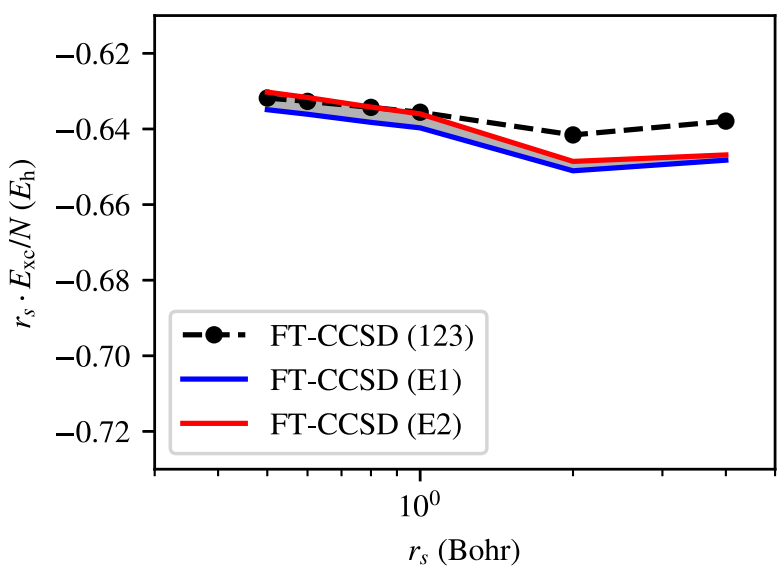

FIG. 14. The FT-CCSD exchange-correlation energy of the $N=33$ polarized UEG in two different plane-wave basis sets at a reduced temperature of $\theta=0.25$. The exchange-correlation energy is scaled by $r_{s}$ to make the scale of the plot more uniform. The solid line is the extrapolated value.

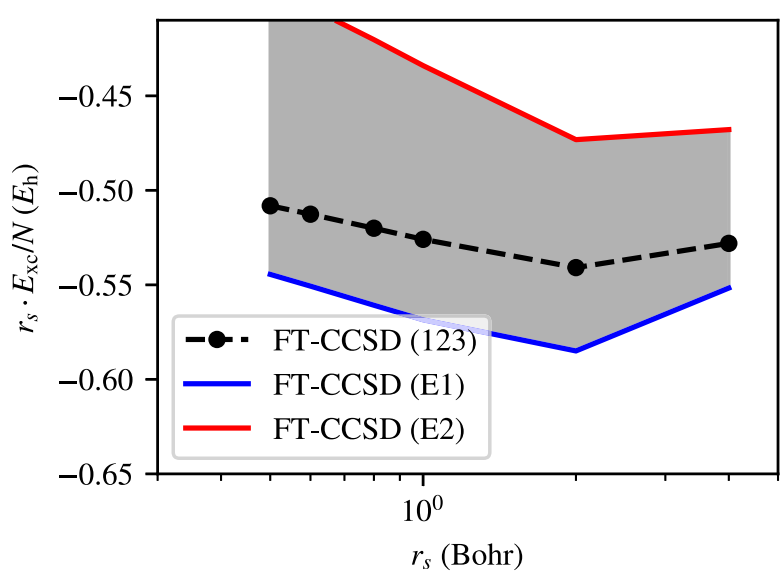

FIG. 15. The FT-CCSD exchange-correlation energy of the $N=66$ unpolarized UEG in a basis set of 123 plane-wave basis orbitals at a reduced temperature of $\theta=0.5$. The exchange-correlation energy is scaled by $r_{s}$ to make the scale of the plot more uniform. E1 and E2 extrapolations are plotted in blue and red, respectively.

In Figs. 15 and 16, we plot the FT-CCSD exchange-correlation energy of the unpolarized UEG in a basis set of 123 plane waves along with the results of E1 and E2 extrapolations.

As with the polarized UEG, there is a larger difference between the E1 and E2 methods at higher temperatures. This makes sense because at higher temperature, states with larger kinetic energy will be thermally populated, and a larger plane-wave basis will be necessary. Unlike for the polarized UEG, the basis set extrapolation is probably not reliable at either temperature. Calculations in larger basis sets should allow for basis set extrapolation with greater confidence.

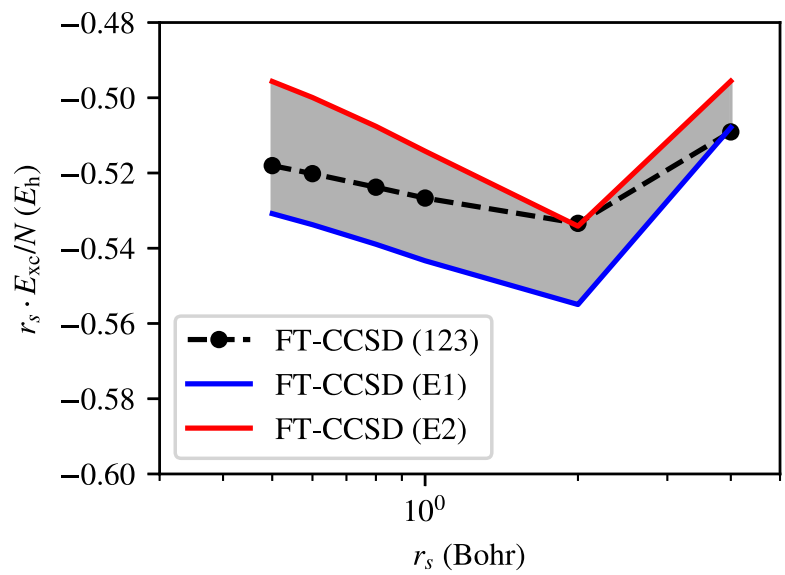

FIG. 16. The FT-CCSD exchange-correlation energy of the $N=66$ polarized UEG in a basis set of 123 plane-wave basis orbitals at a reduced temperature of $\theta=0.25$. The exchange-correlation energy is scaled by $r_{s}$ to make the scale of the plot more uniform. E1 and E2 extrapolations are plotted in blue and red, respectively. 


\section{DATA AVAILABILITY}

The data that support the findings of this study are available within the article [and its supplementary material].

\section{REFERENCES}

${ }^{1}$ V. E. Fortov, Phys.-Usp. 52, 615 (2009).

${ }^{2}$ Frontiers and Challenges in Warm Dense Matter, edited by M. P. Desjarlais, F. Graziani, S. B. Trickey, and R. Redmer (Springer, New York, 2010).

${ }^{3}$ A. Benuzzi-Mounaix, S. Mazevet, A. Ravasio, T. Vinci, A. Denoeud, M. Koenig, N. Amadou, E. Brambrink, F. Festa, A. Levy, M. Harmand, S. Brygoo, G. Huser, V. Recoules, J. Bouchet, G. Morard, F. Guyot, T. D. Resseguier, K. Myanishi, N. Ozaki, F. Dorchies, J. Gaudin, P. M. Leguay, O. Peyrusse, O. Henry, D. Raffestin, S. L. Pape, R. Smith, and R. Musella, Phys. Scr. T161, 014060 (2014).

${ }^{4}$ R. Ernstorfer, M. Harb, C. T. Hebeisen, G. Sciaini, T. Dartigalongue, and R. J. D. Miller, Science 323, 1033 (2009).

${ }^{5}$ L. B. Fletcher, H. J. Lee, T. Döppner, E. Galtier, B. Nagler, P. Heimann, C. Fortmann, S. LePape, T. Ma, M. Millot, A. Pak, D. Turnbull, D. A. Chapman, D. O. Gericke, J. Vorberger, T. White, G. Gregori, M. Wei, B. Barbrel, R. W. Falcone, C. C. Kao, H. Nuhn, J. Welch, U. Zastrau, P. Neumayer, J. B. Hastings, and S. H. Glenzer, Nat. Photonics 9, 274 (2015).

${ }^{6}$ C. Wang, X. T. He, and P. Zhang, Phys. Plasmas 18, 16 (2011); arXiv:1101.4793. ${ }^{7}$ S. X. Hu, L. A. Collins, V. N. Goncharov, J. D. Kress, R. L. McCrory, and S. Skupsky, Phys. Rev. E 92, 043104 (2015).

${ }^{8}$ S. Zhang, K. P. Driver, F. Soubiran, and B. Militzer, Phys. Rev. E 96, 013204 (2017); arXiv:1706.09073.

${ }^{9}$ F. Perrot and M. W. C. Dharma-Wardana, Phys. Rev. A 30, 2619 (1984).

${ }^{10}$ V. V. Karasiev, T. Sjostrom, J. Dufty, and S. B. Trickey, Phys. Rev. Lett. 112, 076403 (2014)

${ }^{11}$ V. V. Karasiev, L. Calderín, and S. B. Trickey, Phys. Rev. E 93, 063207 (2016).

${ }^{12}$ D. M. Ceperley, J. Stat. Phys. 63, 1237 (1991).

${ }^{13}$ W. M. C. Foulkes, L. Mitas, R. J. Needs, and G. Rajagopal, Rev. Mod. Phys. 73, 33 (2001).

${ }^{14}$ T. Schoof, M. Bonitz, A. Filinov, D. Hochstuhl, and J. W. Dufty, Contrib. Plasma Phys. 51, 687 (2011).

${ }^{15}$ N. S. Blunt, T. W. Rogers, J. S. Spencer, and W. M. C. Foulkes, Phys. Rev. B 89, 245124 (2014); arXiv:1303.5007.

${ }^{16}$ T. Dornheim, S. Groth, A. Filinov, and M. Bonitz, New J. Phys. 17, 073017 (2015).

${ }^{17}$ Y. Liu, M. Cho, and B. Rubenstein, J. Chem. Theory Comput. 14, 4722 (2018); arXiv:1806.02848v1.

${ }^{18}$ Y. Liu, T. Shen, H. Zhang, and B. Rubenstein, e-print arXiv:2004.01194 (2020), p. 1.

${ }^{19}$ E. W. Brown, B. K. Clark, J. L. Dubois, and D. M. Ceperley, Phys. Rev. Lett. 110, 146405 (2013); arXiv:1211.6130.

${ }^{20}$ E. W. Brown, J. L. Dubois, M. Holzmann, and D. M. Ceperley, Phys. Rev. B 88, 081102 (2013); arXiv:1306.1863v1.

${ }^{21}$ T. Sjostrom and J. Dufty, Phys. Rev. B 88, 115123 (2013); arXiv:1308.2021.

${ }^{22}$ V. S. Filinov, V. E. Fortov, M. Bonitz, and Z. Moldabekov, Phys. Rev. E 91 , 033108 (2015); arXiv: 1407.3600.

${ }^{23}$ T. Schoof, S. Groth, J. Vorberger, and M. Bonitz, Phys. Rev. Lett. 115, 130402 (2015); arXiv:1502.04616v2.

${ }^{24}$ T. Schoof, S. Groth, and M. Bonitz, Contrib. Plasma Phys. 55, 136 (2015); arXiv:1409.6534.

${ }^{25}$ S. Groth, T. Schoof, T. Dornheim, and M. Bonitz, Phys. Rev. B 93, 085102 (2016).

${ }^{26}$ F. D. Malone, N. S. Blunt, E. W. Brown, D. K. Lee, J. S. Spencer, W. M. Foulkes, and J. J. Shepherd, Phys. Rev. Lett. 117, 115701 (2016); arXiv:1602.05104.

${ }^{27}$ T. Dornheim, S. Groth, T. Schoof, C. Hann, and M. Bonitz, Phys. Rev. B 93, 205134 (2016); arXiv:1601.04977.

${ }^{28}$ T. Dornheim, S. Groth, T. Sjostrom, F. D. Malone, W. M. Foulkes, and M. Bonitz, Phys. Rev. Lett. 117, 156403 (2016); arXiv:1607.08076.
${ }^{29}$ T. Dornheim, S. Groth, and M. Bonitz, Phys. Rep. 744, 1 (2018); arXiv:1801.05783v1.

${ }^{30}$ J. Hubbard, Proc. R. Soc. London, Ser. A 276, 238 (1963).

${ }^{31}$ K. Haule and G. Kotliar, Phys. Rev. B 76, 104509 (2007); arXiv:0709.0019.

${ }^{32}$ S. X. Yang, H. Fotso, S. Q. Su, D. Galanakis, E. Khatami, J. H. She, J. Moreno, J. Zaanen, and M. Jarrell, Phys. Rev. Lett. 106, 047004 (2011); arXiv:1101.6050.

${ }^{33}$ E. Gull, O. Parcollet, and A. J. Millis, Phys. Rev. Lett. 110, 216405 (2013); arXiv:1207.2490.

${ }^{34}$ J. P. F. LeBlanc, A. E. Antipov, F. Becca, I. W. Bulik, G. K.-L. Chan, C.-M. Chung, Y. Deng, M. Ferrero, T. M. Henderson, C. A. Jiménez-Hoyos, E. Kozik, X.-W. Liu, A. J. Millis, N. V. Prokofev, M. Qin, G. E. Scuseria, H. Shi, B. V. Svistunov, L. F. Tocchio, I. S. Tupitsyn, S. R. White, S. Zhang, B.-X. Zheng, Z. Zhu, and E. Gull, Phys. Rev. X 5, 041041 (2015); arXiv:1505.02290.

${ }^{35}$ A. Georges, G. Kotliar, W. Krauth, and M. Rozenberg, Rev. Mod. Phys. 68, 13 (1996).

${ }^{36}$ G. Kotliar, S. Y. Savrasov, K. Haule, V. S. Oudovenko, O. Parcollet, and C. A. Marianetti, Rev. Mod. Phys. 78, 865 (2006); arXiv:0511085 [cond-mat].

${ }^{37}$ M. Hettler, A. Tahvildar-Zadeh, M. Jarrell, and T. Pruschke, Phys. Rev. B 58, R7475 (1998); arXiv:9803295 [cond-mat].

${ }^{38}$ M. Jarrell, T. Maier, M. H. Hettler, and A. N. Tahvildarzadeh, Europhys. Lett. 56, 563 (2001); arXiv:0011282 [cond-mat].

${ }^{39}$ X. Wang and T. Xiang, Phys. Rev. B 56, 5061 (1997).

${ }^{40}$ F. Verstraete, J. J. Garcia-Ripoll, and J. I. Cirac, Phys. Rev. Lett. 93, 207204 (2004); arXiv:0406426 [cond-mat]

${ }^{41}$ S. R. White, Phys. Rev. Lett. 102, 190601 (2009); arXiv:0902.4475.

${ }^{42}$ K. Doll, N. M. Harrison, and V. R. Saunders, J. Phys.: Condens. Matter 11, 5007 (1999); arXiv:9907031 [cond-mat].

${ }^{43}$ J. Aarons, M. Sarwar, D. Thompsett, and C.-K. Skylaris, J. Chem. Phys. 145, 220901 (2016).

${ }^{44}$ P. Liu, M. Kaltak, J. Klimeš, and G. Kresse, Phys. Rev. E 94, 165109 (2016); arXiv:1607.02859.

${ }^{45}$ N. D. Mermin, Ann. Phys. 21, 99 (1963).

${ }^{46}$ N. D. Mermin, Phys. Rev. 137, 1441 (1965); arXiv:1011.1669v3.

${ }^{47}$ Z. Kou and S. Hirata, Theor. Chem. Acc. 133, 1487 (2014).

${ }^{48}$ M. R. Hermes and S. Hirata, J. Chem. Phys. 143, 102818 (2015).

${ }^{49}$ R. Santra and J. Schirmer, Chem. Phys. 482, 355 (2017).

${ }^{50}$ G. Harsha, T. M. Henderson, and G. E. Scuseria, J. Chem. Phys. 150, 154109 (2019); arXiv:1901.06753.

${ }^{51}$ A. A. Kananenka, A. R. Welden, T. N. Lan, E. Gull, and D. Zgid, J. Chem. Theory Comput. 12, 2250 (2016); arXiv:1602.05898.

${ }^{52}$ A. R. Welden, A. A. Rusakov, and D. Zgid, J. Chem. Phys. 145, 204106 (2016); arXiv:1605.06563.

${ }^{53}$ D. Neuhauser, R. Baer, and D. Zgid, J. Chem. Theory Comput. 13, 5396 (2017); arXiv:1707.08296.

${ }^{54}$ S. H. Mandal, R. Ghosh, G. Sanyal, and D. Mukherjee, Int. J. Mod. Phys. B 17, 5367 (2003).

${ }^{55}$ A. F. White and G. Chan, J. Chem. Theory Comput. 14, 5690 (2018); arXiv:1807.09961.

${ }^{56}$ F. Hummel, J. Chem. Theory Comput. 14, 6505 (2018).

${ }^{57}$ G. Harsha, T. M. Henderson, and G. E. Scuseria, J. Chem. Theory Comput. 15, 6127 (2019); arXiv:1907.11286.

${ }^{58}$ J. Paldus, J. Čížek, and I. Shavitt, Phys. Rev. A 5, 50 (1972).

${ }^{59}$ J. Cizek and J. Paldus, Phys. Scr. 21, 251 (1980).

${ }^{60}$ R. J. Bartlett, Annu. Rev. Phys. Chem. 32, 359 (1981)

${ }^{61}$ G. D. Purvis and R. J. Bartlett, J. Chem. Phys. 76, 1910 (1982).

${ }^{62}$ T. D. Crawford and H. F. Schaefer III, in Reviews in Computational Chemistry, edited by K. B. Lipkowitz and D. B. Boyd (John Wiley \& Sons, Inc., Hoboken, NJ, USA, 2000), Vol. 14.

${ }^{63}$ R. J. Bartlett and M. Musiał, Rev. Mod. Phys. 79, 291 (2007).

${ }^{64}$ I. Shavitt and R. J. Bartlett, Many-Body Methods in Chemistry and Physics: $M B P T$ and Coupled-Cluster Theory (Cambridge University Press, New York, 2009).

${ }^{65}$ G. Sanyal, S. H. Mandal, and D. Mukherjee, Chem. Phys. Lett. 192, 55 (1992). 
${ }^{66}$ G. Sanyal, S. H. Mandal, S. Guha, and D. Mukherjee, Phys. Rev. E 48, 3373 (1993).

${ }^{67}$ S. H. Mandal, R. Ghosh, and D. Mukherjee, Chem. Phys. Lett. 335, 281 (2001).

${ }^{68}$ S. H. Mandal, R. Ghosh, G. Sanyal, and D. Mukherjee, Chem. Phys. Lett. 352, 63 (2002).

${ }^{69}$ A. A. Dzhioev and D. S. Kosov, J. Phys. A: Math. Theor. 48, 015004 (2015).

${ }^{70}$ A. F. White and G. K. L. Chan, J. Chem. Theory Comput. 15, 6137 (2019); arXiv:1907.11695.

${ }^{71}$ P. Shushkov and T. F. Miller, J. Chem. Phys. 151, 134107 (2019); arXiv:1907.11962.

${ }^{72}$ G. Sanyal, S. H. Mandal, and D. Mukherjee, Proc. Indian Acad. Sci. 106, 407 (1994), see https://link.springer.com/article/10.1007/BF02840760.

${ }^{73}$ C. Runge, Math. Ann. 46, 167 (1895).

${ }^{74} \mathrm{E}$. W. Weisstein, Runge-Kutta Method, http://mathworld.wolfram.com/RungeKuttaMethod.html; accessed 25 February 2020.

${ }^{75} \mathrm{~F}$. Bashforth and J. C. Adams, Theories of Capillary Action (Cambridge University Press, London, 1883).

${ }^{76} \mathrm{E}$. W. Weisstein, Adams' Method, https://mathworld.wolfram.com/ AdamsMethod.html; accessed 25 February 2020.

${ }^{77}$ J. Crank and P. Nicolson, Math. Proc. Cambridge Philos. Soc. 43, 50 (1947).

${ }^{78}$ E. W. Weisstein, Simpson's Rule, http://mathworld.wolfram.com/ SimpsonsRule.html; accessed 25 February 2020.

${ }^{79}$ M. Takahashi and M. Shiroishi, Phys. Rev. B 65, 165104 (2002).

${ }^{80}$ J. Paldus, M. Takahashi, and R. W. Cho, Phys. Rev. B 30, 4267 (1984).

${ }^{81}$ Y. Asai and H. Katagiri, Phys. Rev. B 60, R13946 (1999).

${ }^{82}$ N. D. Mermin and H. Wagner, Phys. Rev. Lett. 17, 1133 (1966).
${ }^{83}$ T. Dornheim, T. Schoof, S. Groth, A. Filinov, and M. Bonitz, J. Chem. Phys. 143, 204101 (2015); arXiv:1508.03221.

${ }^{84}$ T. Dornheim, S. Groth, A. V. Filinov, and M. Bonitz, J. Chem. Phys. 151, 014108 (2019); arXiv:1902.06741.

${ }^{85}$ V. A. Neufeld and A. J. Thom, J. Chem. Phys. 147, 194105 (2017); arXiv:1706.09923.

${ }^{86}$ P. F. Loos and P. M. Gill, Wiley Interdiscip. Rev.: Comput. Mol. Sci. 6, 410 (2016); arXiv:1601.03544.

${ }^{87} \mathrm{~J}$. VandeVondele and J. Hutter, J. Chem. Phys. 127, 114105 (2007).

${ }^{88}$ S. Goedecker and M. Teter, Phys. Rev. B 54, 1703 (1996); arXiv:9512004 [mtrlth].

${ }^{89}$ C. Hartwigsen, S. Goedecker, and J. Hutter, Phys. Rev. B 58, 3641 (1998); arXiv:9803286 [cond-mat].

${ }^{90}$ Q. Sun, T. C. Berkelbach, N. S. Blunt, G. H. Booth, S. Guo, Z. Li, J. Liu, J. D. McClain, E. R. Sayfutyarova, S. Sharma, S. Wouters, and G. K. L. Chan, Wiley Interdiscip. Rev.: Comput. Mol. Sci. 8, e1340 (2018); arXiv:1701.08223.

${ }^{91} \mathrm{~J}$. VandeVondele, M. Krack, F. Mohamed, M. Parrinello, T. Chassaing, and J. Hutter, Comput. Phys. Commun. 167, 103 (2005).

${ }^{92}$ J. McClain, Q. Sun, G. K. L. Chan, and T. C. Berkelbach, J. Chem. Theory Comput. 13, 1209 (2017); arXiv:1707.07114.

${ }^{93}$ C. Lin, F. H. Zong, and D. M. Ceperley, Phys. Rev. E 64, 016702 (2001).

${ }^{94}$ E. G. Moroni, G. Grimvall, and T. Jarlborg, Phys. Rev. Lett. 76, 2758 (1996).

${ }^{95}$ J. F. Stanton, J. Gauss, J. D. Watts, and R. J. Bartlett, J. Chem. Phys. 94, 4334 (1991); arXiv:1707.04192.

${ }^{96}$ J. Gauss and J. F. Stanton, J. Chem. Phys. 103, 3561 (1995).

${ }^{97}$ E. A. Salter, G. W. Trucks, and R. J. Bartlett, J. Chem. Phys. 90, 1752 (1989). 